Supporting Information

\title{
A critical role of non-classical intermolecular hydrogen bonding in affecting the $\pi-\pi$ stacking and nonlinear optical properties of tricyanofuran-based push-pull heptamethines
}

\author{
Di Zhang, ${ }^{a, b}$ Weilong Chen, ${ }^{a, b}$ Jie Zou, ${ }^{a, b}$ \& Jingdong Luo ${ }^{a, b^{*}}$ \\ a Shenzhen Research Institute, City University of Hong Kong, Shenzhen 518057, China \\ ${ }^{b}$ Department of Chemistry, City University of Hong Kong, Kowloon, Hong Kong SAR, China \\ *Corresponding author: E-mail: jingdluo@,cityu.edu.hk
}

\section{Table of Contents}

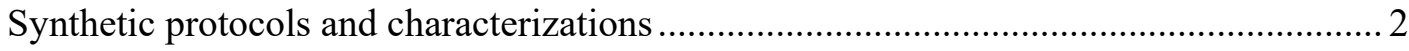

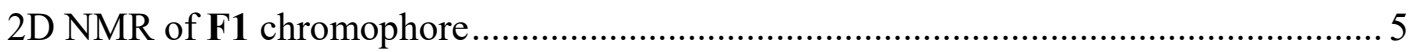

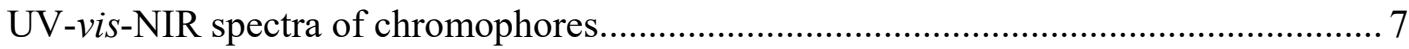

Leak through current profiles of poled films ......................................................... 9

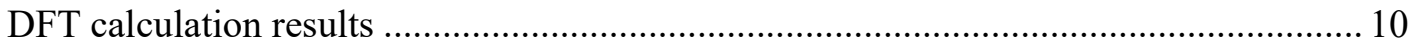

Hydrogen bond geometry of F3 and F3-ON chromophores................................... 12

AFM graphs of F3/P(S-co-MMA) and F3-ON/P(S-co-MMA) films ............................ 13

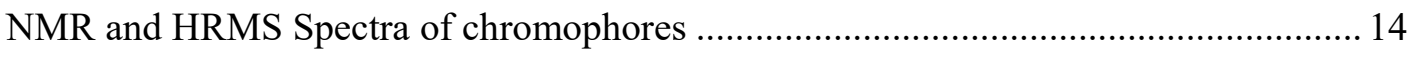

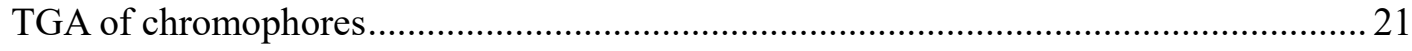

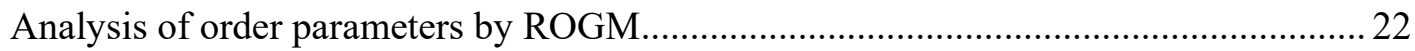

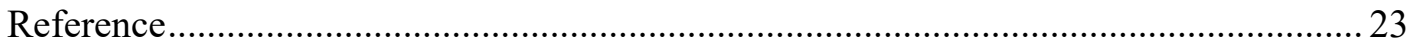


Synthetic protocols and characterizations

\section{Synthesis of F1-ON}

F1 (100 mg, $0.18 \mathrm{mmol})$, 4,5-diphenyloxazole-2-thiol (90 mg, $0.36 \mathrm{mmol})$, triethyamine (40 $\mathrm{mg}, 0.40 \mathrm{mmol}$ ) were added to $10 \mathrm{ml}$ of acetonitrile, and reacted at $65{ }^{\circ} \mathrm{C}$ for 16 hours. The crude product was collected through filtration and rinsed by methanol. Then it was purified by column chromatography by DCM, then DCM/EA (100:1) and recrystallized from DCM/ethanol to afford a dark solid. (125 mg, yield: $90 \%$ \%). ${ }^{1} \mathrm{H}$ NMR (400 MHz, Chloroform-d) $\delta 8.57(\mathrm{~d}, \mathrm{~J}=15.4 \mathrm{~Hz}, 1 \mathrm{H}), 8.46(\mathrm{~d}, \mathrm{~J}=13.0 \mathrm{~Hz}, 1 \mathrm{H}), 7.59-7.52(\mathrm{~m}, 2 \mathrm{H})$, 7.49 - $7.42(\mathrm{~m}, 2 \mathrm{H}), 7.35(\mathrm{~m}, 6 \mathrm{H}), 7.31(1 \mathrm{H}), 7.26(1 \mathrm{H}), 7.07$ (t, J = $7.4 \mathrm{~Hz}, 1 \mathrm{H}), 6.88(\mathrm{~d}, \mathrm{~J}=$ $7.9 \mathrm{~Hz}, 1 \mathrm{H}), 6.58(\mathrm{~d}, \mathrm{~J}=15.4 \mathrm{~Hz}, 1 \mathrm{H}), 5.74(\mathrm{~d}, \mathrm{~J}=13.0 \mathrm{~Hz}, 1 \mathrm{H}), 3.40(\mathrm{~s}, 3 \mathrm{H}), 2.91(2 \mathrm{H}), 2.17$ $(\mathrm{d}, 2 \mathrm{H}), 1.82(\mathrm{~d}, \mathrm{~J}=6.6 \mathrm{~Hz}, 6 \mathrm{H}), 1.70(\mathrm{~d}, \mathrm{~J}=13.5 \mathrm{~Hz}, 6 \mathrm{H}), 1.59(\mathrm{~m}, 1 \mathrm{H}), 1.08(\mathrm{~s}, 9 \mathrm{H}) .{ }^{13} \mathrm{C}$ NMR $\left(101 \mathrm{MHz}, \mathrm{CDCl}_{3}\right) \delta 176.11,173.54,166.18,155.43,148.32,146.00,143.75,137.96$, $136.99,131.54,129.08,128.78,128.62,128.54,128.17,127.71,126.44,122.48,122.00$, 113.23, 112.47, 111.83, 111.45, 108.00, 96.85, 96.11, 94.64, 53.59, 47.51, 42.31, 32.49, 29.98, 28.22, 28.10, 27.42, 27.14, 27.12. HRMS $\left(\mathrm{C}_{50} \mathrm{H}_{47} \mathrm{~N}_{5} \mathrm{O}_{2} \mathrm{~S}\right): \mathrm{m} / \mathrm{z} 781.34485\left(\mathrm{M}^{+}\right.$, calcd $781.34450)$.

\section{Synthesis of F2-ON}

F2 (171.4 mg, $0.26 \mathrm{mmol})$, 4,5-diphenyloxazole-2-thiol (109 $\mathrm{mg}, 0.43 \mathrm{mmol})$, triethyamine (52.8 $\mathrm{mg}, 0.52 \mathrm{mmol}$ ) were added to $10 \mathrm{ml}$ of acetonitrile and $10 \mathrm{ml}$ of chloroform, and reacted at $65{ }^{\circ} \mathrm{C}$ for 16 hours. The crude product was purified by column chromatography (DCM:EA=50:1), and recrystallized from DCM/ethanol to afford a dark solid (174 mg, yield: 76 \%). ${ }^{1} \mathrm{H}$ NMR (300 MHz, Chloroform-d) $\delta 8.73-8.43(\mathrm{~m}, 2 \mathrm{H}), 8.05(\mathrm{~d}, \mathrm{~J}=8.6 \mathrm{~Hz}, 1 \mathrm{H})$, $7.87(\mathrm{t}, \mathrm{J}=9.3 \mathrm{~Hz}, 2 \mathrm{H}), 7.59-7.50(\mathrm{~m}, 3 \mathrm{H}), 7.47(\mathrm{~m}, 2 \mathrm{H}), 7.43-7.31(\mathrm{~m}, 7 \mathrm{H}), 7.20(\mathrm{~d}, \mathrm{~J}=$ $8.8 \mathrm{~Hz}, 1 \mathrm{H}), 6.56(\mathrm{~d}, \mathrm{~J}=15.4 \mathrm{~Hz}, 1 \mathrm{H}), 5.85(\mathrm{~d}, \mathrm{~J}=13.2 \mathrm{~Hz}, 1 \mathrm{H}), 3.94(\mathrm{~s}, 2 \mathrm{H}), 3.08-2.78(\mathrm{~m}$, 2H), $2.18(\mathrm{~m}, 2 \mathrm{H}), 2.00(\mathrm{~d}, \mathrm{~J}=11.8 \mathrm{~Hz}, 6 \mathrm{H}), 1.83(\mathrm{~d}, \mathrm{~J}=5.3 \mathrm{~Hz}, 8 \mathrm{H}), 1.51(\mathrm{~m}, 3 \mathrm{H}), 1.07(\mathrm{~m}$, 12H). HRMS ( $\left.\mathrm{C}_{57} \mathrm{H}_{55} \mathrm{~N}_{5} \mathrm{O}_{2} \mathrm{~S}\right): \mathrm{m} / \mathrm{z} 873.40717\left(\mathrm{M}^{+}\right.$, calcd 873.40710). 


\section{Synthesis of F3-ON}

F3 (162 mg, $0.24 \mathrm{mmol})$, 4,5-diphenyloxazole-2-thiol (100.9 mg, $0.40 \mathrm{mmol}$ ), triethyamine (48.8 $\mathrm{mg}, 0.48 \mathrm{mmol})$ were added to $10 \mathrm{ml}$ of acetonitrile and $10 \mathrm{ml}$ of chloroform, and reacted at $65{ }^{\circ} \mathrm{C}$ for 16 hours. Then it was purified by chromatography with DCM:EA=50:1. Finally, the product was recrystallized from DCM/ethanol to afford a dark solid. (156 mg, yield: $73 \%)^{1} \mathrm{H}$ NMR (300 MHz, Chloroform-d) $\delta 8.82(\mathrm{~d}, \mathrm{~J}=13.0 \mathrm{~Hz}, 1 \mathrm{H}), 8.61(\mathrm{~d}, \mathrm{~J}=15.6$ $\mathrm{Hz}, 1 \mathrm{H}), 8.44(\mathrm{~d}, \mathrm{~J}=7.4 \mathrm{~Hz}, 1 \mathrm{H}), 7.82(\mathrm{~d}, \mathrm{~J}=8.1 \mathrm{~Hz}, 1 \mathrm{H}), 7.65$ (t, J = $7.7 \mathrm{~Hz}, 1 \mathrm{H}), 7.56-$ $7.38(\mathrm{~m}, 6 \mathrm{H}), 7.38-7.29(\mathrm{~m}, 4 \mathrm{H}), 7.28-7.21(\mathrm{~m}, 2 \mathrm{H}), 6.79(\mathrm{~d}, \mathrm{~J}=7.1 \mathrm{~Hz}, 1 \mathrm{H}), 6.68(\mathrm{~d}, \mathrm{~J}=$ $15.6 \mathrm{~Hz}, 1 \mathrm{H}), 6.31(\mathrm{~d}, \mathrm{~J}=13.1 \mathrm{~Hz}, 1 \mathrm{H}), 3.88(\mathrm{~d}, \mathrm{~J}=7.2 \mathrm{~Hz}, 2 \mathrm{H}), 3.09$ (d, J = 14.8 Hz, 1H), $2.90(\mathrm{~d}, \mathrm{~J}=15.5 \mathrm{~Hz}, 1 \mathrm{H}), 2.39$ - $2.10(\mathrm{~m}, 2 \mathrm{H}), 2.06-1.93(\mathrm{~m}, 1 \mathrm{H}), 1.85(\mathrm{~s}, 6 \mathrm{H}), 1.67(\mathrm{~s}, 1 \mathrm{H})$, $1.53-1.26(\mathrm{~m}, 8 \mathrm{H}), 1.09$ (s, 9H), $1.00(\mathrm{~m}, 3 \mathrm{H}), 0.90(\mathrm{~m}, 3 \mathrm{H}) .{ }^{13} \mathrm{C} \mathrm{NMR}\left(101 \mathrm{MHz}, \mathrm{CDCl}_{3}\right) \delta$ $175.81,173.61,155.23,148.46,148.20,145.87,143.62,142.31,138.92,137.13,135.35$, $131.48,131.11,130.60,129.36,129.09,128.92,128.73,128.59,128.54,128.09,127.73$, $127.48,126.48,126.41,124.42,118.31,113.47,112.80,112.05,111.04,103.69,103.37,97.15$, $96.56,54.84,47.57,42.35,39.23,32.50,31.39,31.05,29.21,29.07,27.89,27.36,27.09$, 27.04, 24.55, 24.36, 23.12, 14.05, 11.20. HRMS $\left(\mathrm{C}_{58} \mathrm{H}_{57} \mathrm{~N}_{5} \mathrm{O}_{2} \mathrm{~S}\right): \mathrm{m} / \mathrm{z} 887.42297\left(\mathrm{M}^{+}\right.$, calcd $887.42275)$.

\section{Synthesis of M1-ON}

M1 (124.3 mg, $0.17 \mathrm{mmol})$, 4,5-diphenyloxazole-2-thiol (72.73 mg, $0.29 \mathrm{mmol})$, triethyamine (35.2 mg, $0.35 \mathrm{mmol}$ ) were mixed in $10 \mathrm{ml}$ of acetonitrile, and reacted at $65^{\circ} \mathrm{C}$ for 18 hours. It was purified by column chromatography (DCM:EA=50:1), and recrystallized from DCM/ethanol to afford a dark solid. (98 mg, yield: $60 \%$ \% ${ }^{1} \mathrm{H}$ NMR (300 MHz, Chloroform-d) $\delta 8.45(\mathrm{~d}, \mathrm{~J}=15.7 \mathrm{~Hz}, 1 \mathrm{H}), 7.93(\mathrm{~d}, \mathrm{~J}=12.1 \mathrm{~Hz}, 1 \mathrm{H}), 7.66-7.55(\mathrm{~m}, 2 \mathrm{H}), 7.55-7.47(\mathrm{~m}, 2 \mathrm{H})$, 7.43 - $7.35(\mathrm{~m}, 6 \mathrm{H}), 7.35$ - $7.30(\mathrm{~m}, 2 \mathrm{H}), 7.16$ - $7.05(\mathrm{~m}, 2 \mathrm{H}), 6.88(\mathrm{~d}, \mathrm{~J}=12.2 \mathrm{~Hz}, 1 \mathrm{H}), 6.73$ $6.56(\mathrm{~m}, 3 \mathrm{H}), 6.47(\mathrm{~d}, \mathrm{~J}=8.8 \mathrm{~Hz}, 2 \mathrm{H}), 3.43(\mathrm{q}, \mathrm{J}=7.0 \mathrm{~Hz}, 4 \mathrm{H}), 3.22(\mathrm{~m}, 5 \mathrm{H}), 2.82$ (d, J = $16.4 \mathrm{~Hz}, 1 \mathrm{H}), 2.40-2.11(\mathrm{~m}, 2 \mathrm{H}), 1.77(\mathrm{~d}, 6 \mathrm{H}), 1.57(\mathrm{~s}, 1 \mathrm{H}), 1.24(\mathrm{q}, 6 \mathrm{H}), 1.07(\mathrm{~m}, 15 \mathrm{H}) .{ }^{13} \mathrm{C}$ NMR $\left(100 \mathrm{MHz}, \mathrm{CDCl}_{3}\right) \delta 175.70,173.91,155.96,153.81,148.56,148.26,147.87,145.73$, 
$139.25,136.83,133.13,131.69,131.00,129.54,128.96,128.87,128.68,128.55,128.33$, $127.77,126.32,125.73,119.64,113.93,112.59,111.82,110.98,110.76,110.62,97.33,55.35$, $44.50,44.24,42.63,32.54,28.57,28.39,27.39,27.01,26.95,12.70,12.61$. HRMS $\left(\mathrm{C}_{60} \mathrm{H}_{62} \mathrm{~N}_{6} \mathrm{O}_{2} \mathrm{~S}\right): \mathrm{m} / \mathrm{z} 930.46545\left(\mathrm{M}^{+}\right.$, calcd 930.46495).

\section{Synthesis of M2-ON}

M2 (100 mg, $0.16 \mathrm{mmol}), 4,5$-diphenyloxazole-2-thiol (65 mg, $0.26 \mathrm{mmol})$, triethyamine (30 $\mathrm{mg}, 0.30 \mathrm{mmol}$ ) were reacted in acetonitrile at $65{ }^{\circ} \mathrm{C}$ for 20 hours. The crude product was rinsed by methanol, purified by flash chroma-tography (DCM:EA=100:1), and recrystallized with DCM/Ethanol. (125 mg, yield: $94 \%$ ) ${ }^{1} \mathrm{H}$ NMR (400 MHz, Chloroform-d) $\delta 7.86$ (d, J = $15.6 \mathrm{~Hz}, 1 \mathrm{H}), 7.76$ - 7.59 (m, 2H), 7.59 - 7.49 (m, 2H), 7.39 (m, 6H), 7.35 - 7.29 (m, 3H), 7.18 - $7.02(\mathrm{~m}, 2 \mathrm{H}), 6.77$ - $6.60(\mathrm{~m}, 3 \mathrm{H}), 6.59$ - $6.45(\mathrm{~m}, 3 \mathrm{H}), 3.43(\mathrm{q}, \mathrm{J}=7.0 \mathrm{~Hz}, 4 \mathrm{H}), 3.23$ (q, $\mathrm{J}=7.0 \mathrm{~Hz}, 4 \mathrm{H}), 3.04(\mathrm{~d}, \mathrm{~J}=45.2 \mathrm{~Hz}, 4 \mathrm{H}), 1.72(\mathrm{~s}, 6 \mathrm{H}), 1.22(\mathrm{t}, \mathrm{J}=7.0 \mathrm{~Hz}, 6 \mathrm{H}), 1.08$ (t, J = $7.0 \mathrm{~Hz}, 6 \mathrm{H}) .{ }^{13} \mathrm{C} \mathrm{NMR}\left(100 \mathrm{MHz}, \mathrm{CDCl}_{3}\right) \delta 175.53,172.64,154.64,152.00,148.46,148.23$, $148.13,146.05,144.52$, 138.56, 136.96, 132.87, 131.53, 131.39, 130.81, 129.51, 129.08, $128.89,128.73,128.61,128.18,127.76,126.45,125.62,121.01,115.52,112.52,111.75$, $111.00,110.80,110.59,97.57,97.04,55.56,44.50,44.17,29.21,27.19,26.90,12.70,12.64$. HRMS $\left(\mathrm{C}_{55} \mathrm{H}_{52} \mathrm{~N}_{6} \mathrm{O}_{2} \mathrm{~S}\right): \mathrm{m} / \mathrm{z} 883.37616\left([\mathrm{M}+\mathrm{Na}]^{+}\right.$, calcd 883.37647). 
2D NMR of $\mathbf{F 1}$ chromophore

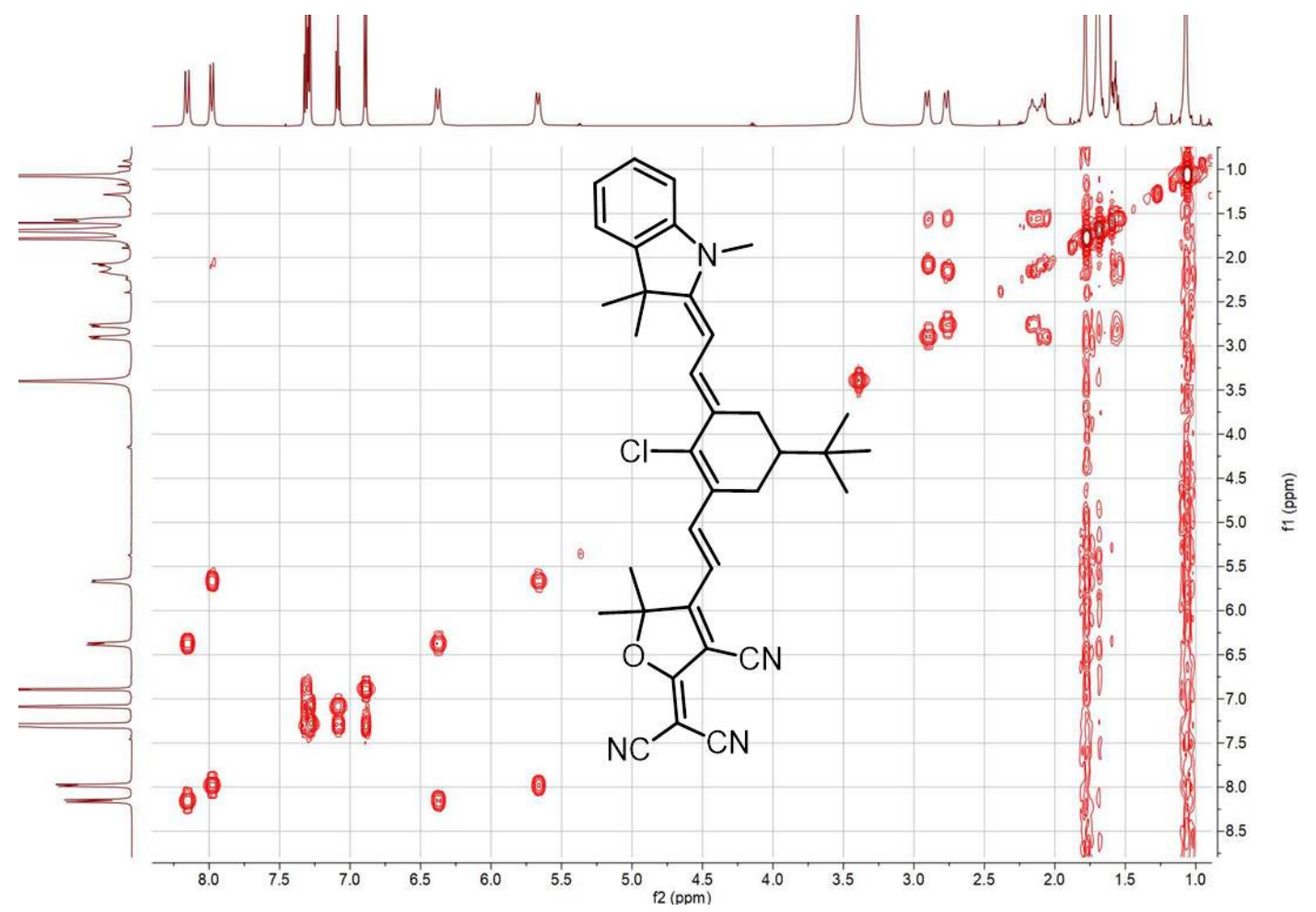

Fig. S1 COSY spectrum of the F1 chromophore.

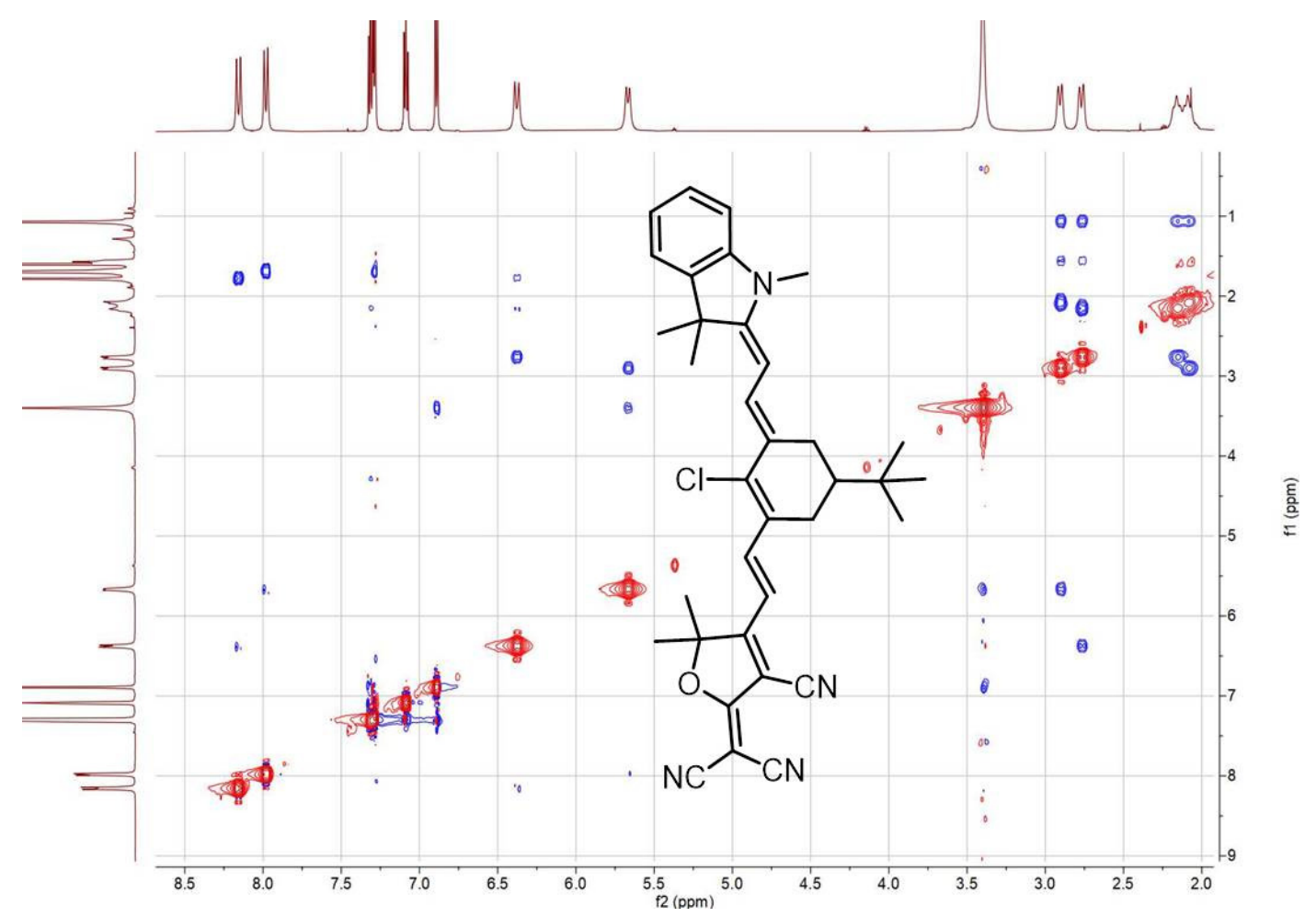

Fig. S2 NOESY spectrum of the F1 chromophore. 
Table S1. Chemical shifts and spin-spin coupling constants ${ }^{3} J(\mathrm{H}, \mathrm{H})$ of hydrogens in the polymethine chain of chromophores.

\begin{tabular}{c|cc|cc|ccc|cc}
\hline \multirow{2}{*}{ Chromophore } & \multicolumn{2}{|c|}{$\mathrm{a}$} & \multicolumn{2}{c|}{$\mathrm{b}$} & \multicolumn{2}{c|}{$\mathrm{c}$} & \multicolumn{2}{c}{$\mathrm{d}$} \\
\cline { 2 - 9 } & $\delta / \mathrm{ppm}$ & $J / \mathrm{Hz}$ & $\delta / \mathrm{ppm}$ & $J / \mathrm{Hz}$ & $\delta / \mathrm{ppm}$ & $J / \mathrm{Hz}$ & $\delta / \mathrm{ppm}$ & $J / \mathrm{Hz}$ \\
\hline F1 & 5.68 & 13.2 & 8.00 & 13.2 & 8.15 & 15.2 & 6.36 & 15.4 \\
F1-ON & 5.74 & 13.0 & 8.46 & 13.0 & 8.57 & 15.4 & 6.58 & 15.4 \\
F2 & 5.79 & 13.4 & & - & & & 6.35 & 15.2 \\
F2-ON & 5.85 & 13.2 & & - & & - & 6.56 & 15.4 \\
F3 & 6.22 & 13.1 & 8.30 & 13.0 & 8.19 & 15.5 & 6.46 & 15.5 \\
F3-ON & 6.31 & 13.1 & 8.83 & 12.9 & 8.62 & 15.6 & 6.68 & 15.6 \\
M1 & 6.81 & 12.2 & 7.52 & 12.2 & 8.10 & 15.7 & 6.50 & 15.7 \\
M1-ON & 6.88 & 12.2 & 7.93 & 12.1 & 8.47 & 15.7 & \multicolumn{2}{|c}{-} \\
M2 & & & 7.08 & 12.5 & 7.66 & 15.6 & 6.37 & 15.7 \\
M2-ON & 6.71 & 12.4 & & - & 6.86 & 15.6 & \multicolumn{2}{|c}{-} \\
\hline
\end{tabular}


UV-vis-NIR spectra of chromophores
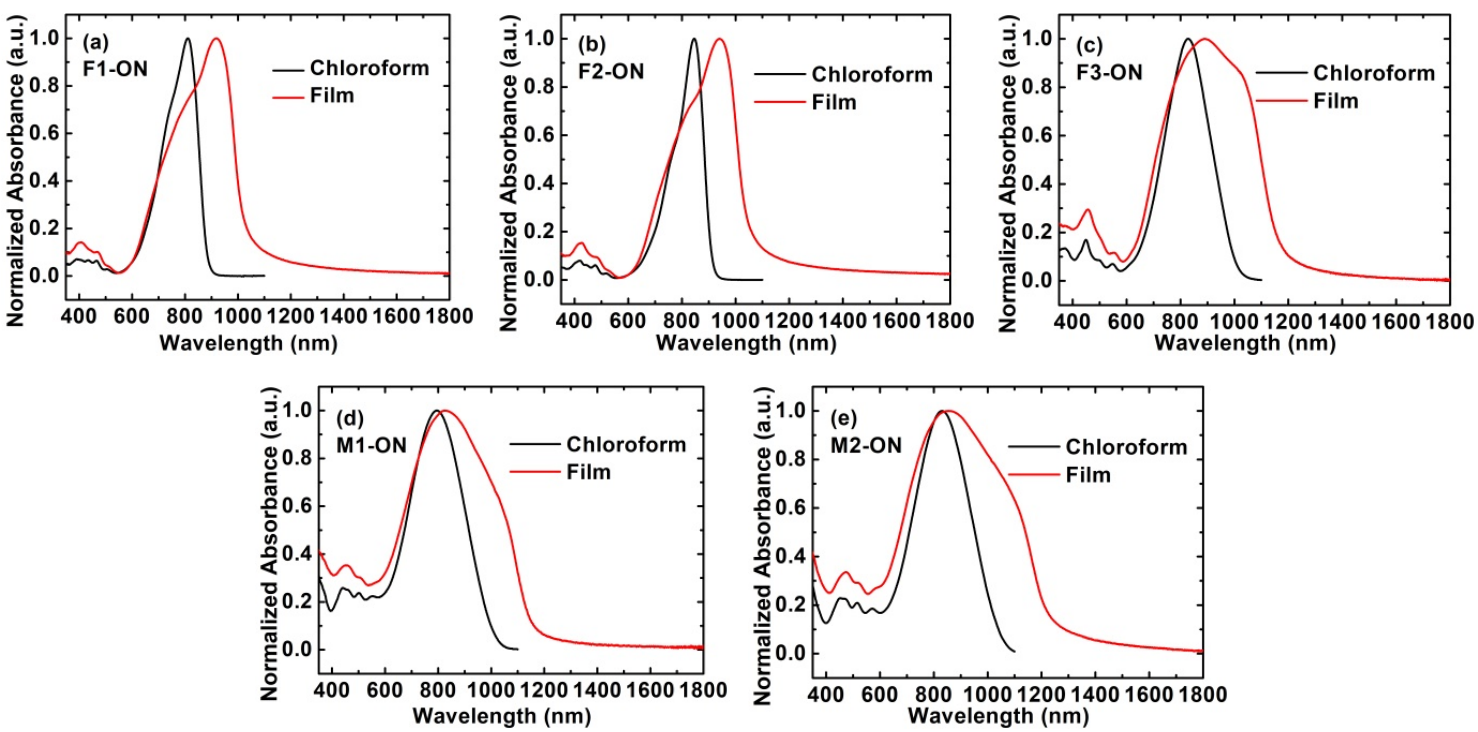

Fig. S3 UV-vis-NIR spectra of five compounds in the $\mathrm{CHCl}_{3}$ solution and the net film.

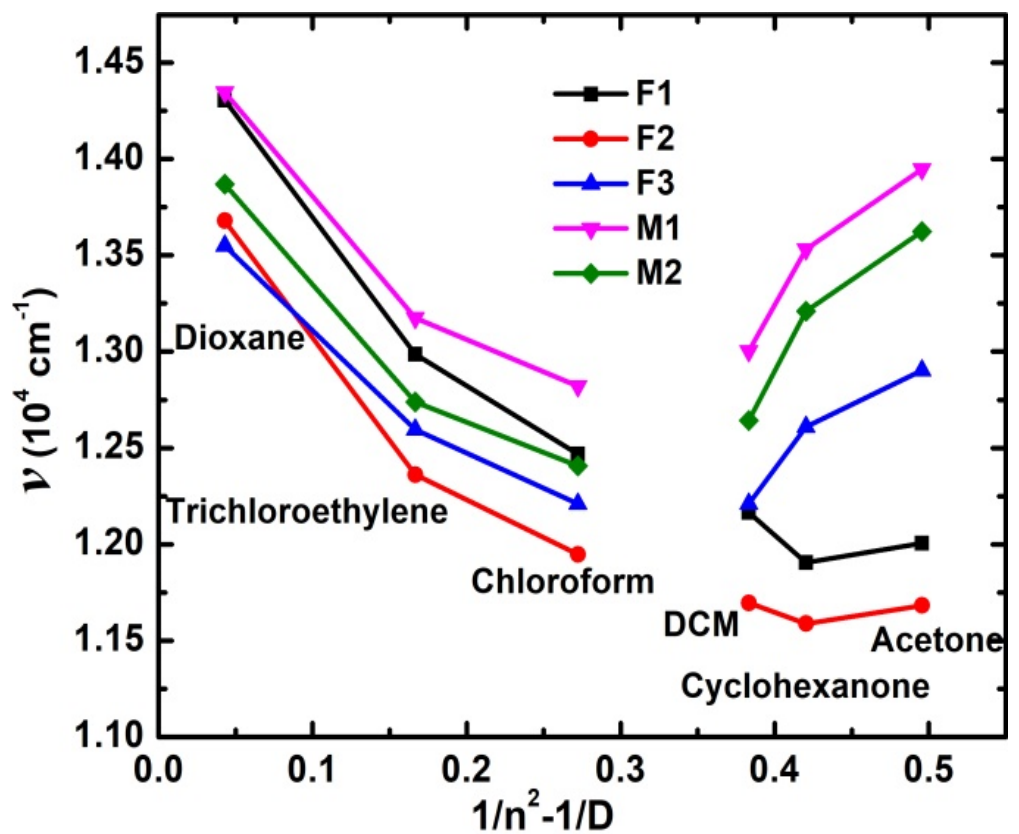

Fig. S4 Hush approach in analyzing the dependence of transition energy $v$ in $\mathrm{cm}^{-1}$ on solvent polarization for chloro-substituted heptamethines. 


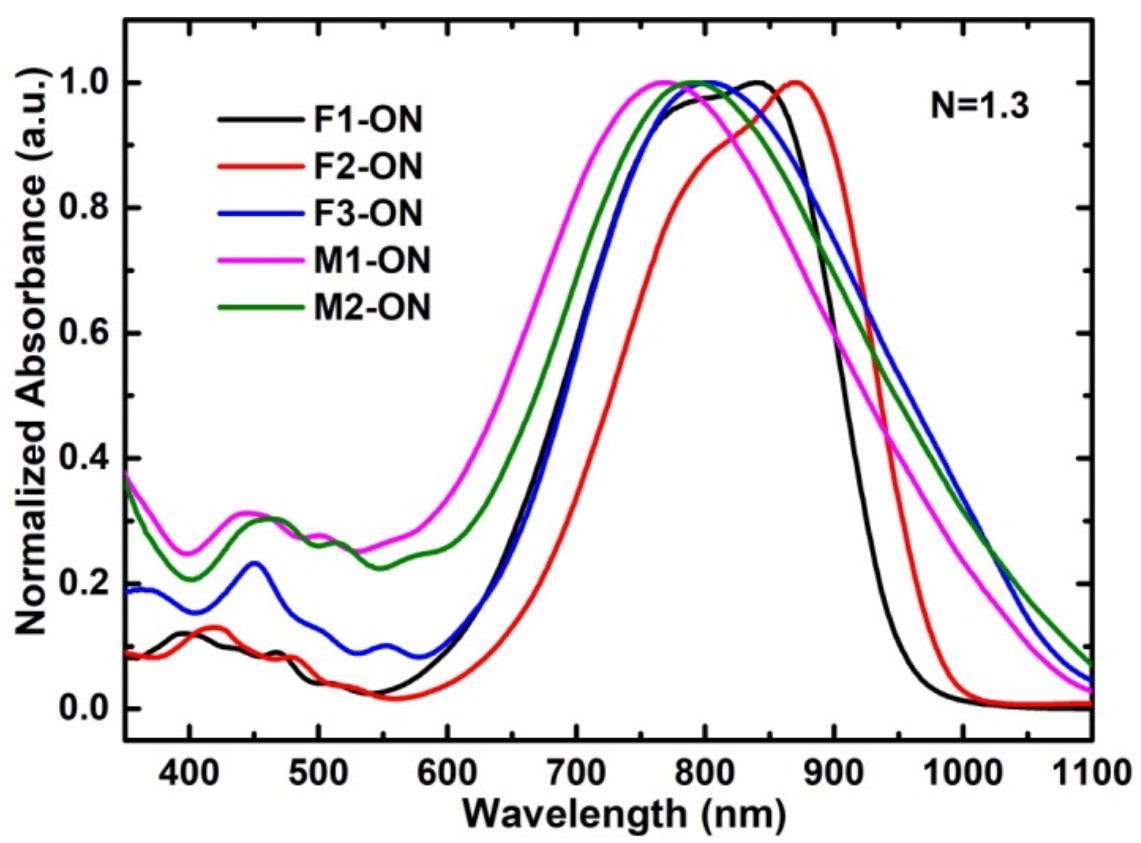

Fig. S5 UV-vis-NIR spectra of thin films by doping the thioether-substituted chromophores into the polymer $\mathrm{P}(\mathrm{S}-\mathrm{co}-\mathrm{MMA})$ at a number density of $1.3 \times 10^{20} \mathrm{~cm}^{3}$. 
Leak through current profiles of poled films

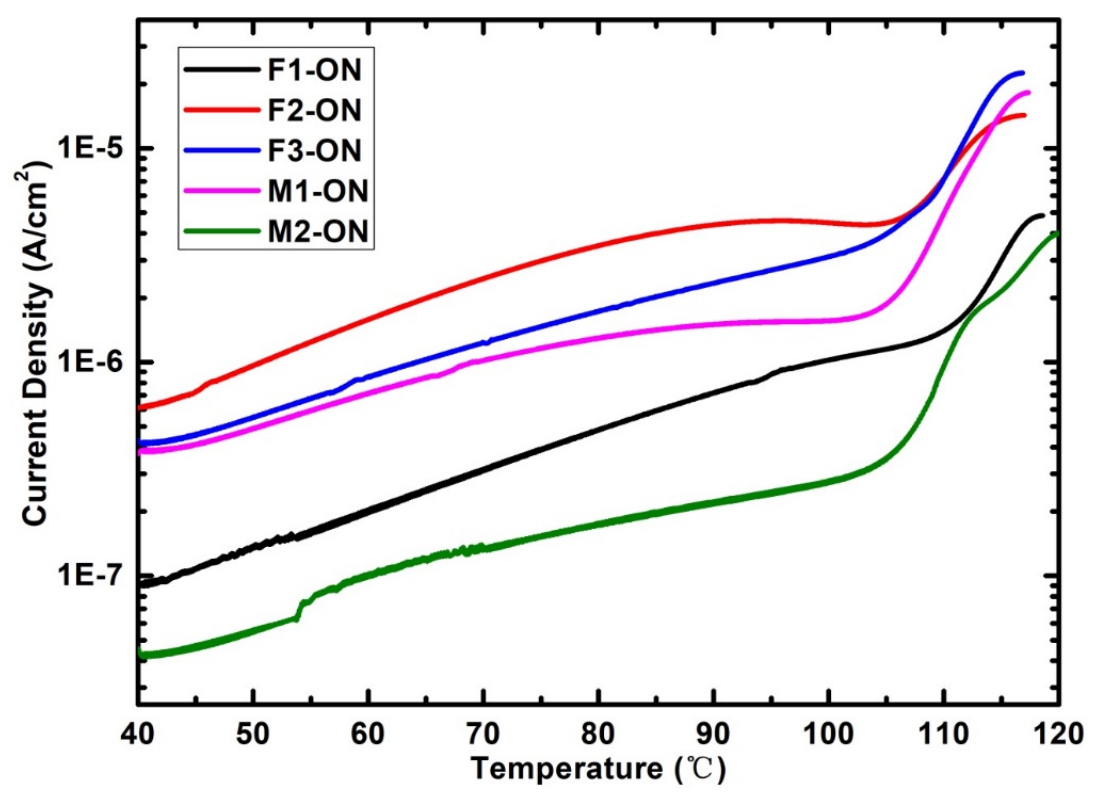

Fig. S6 Leak through current profile with the change of temperatures for poled films. 
DFT calculation results
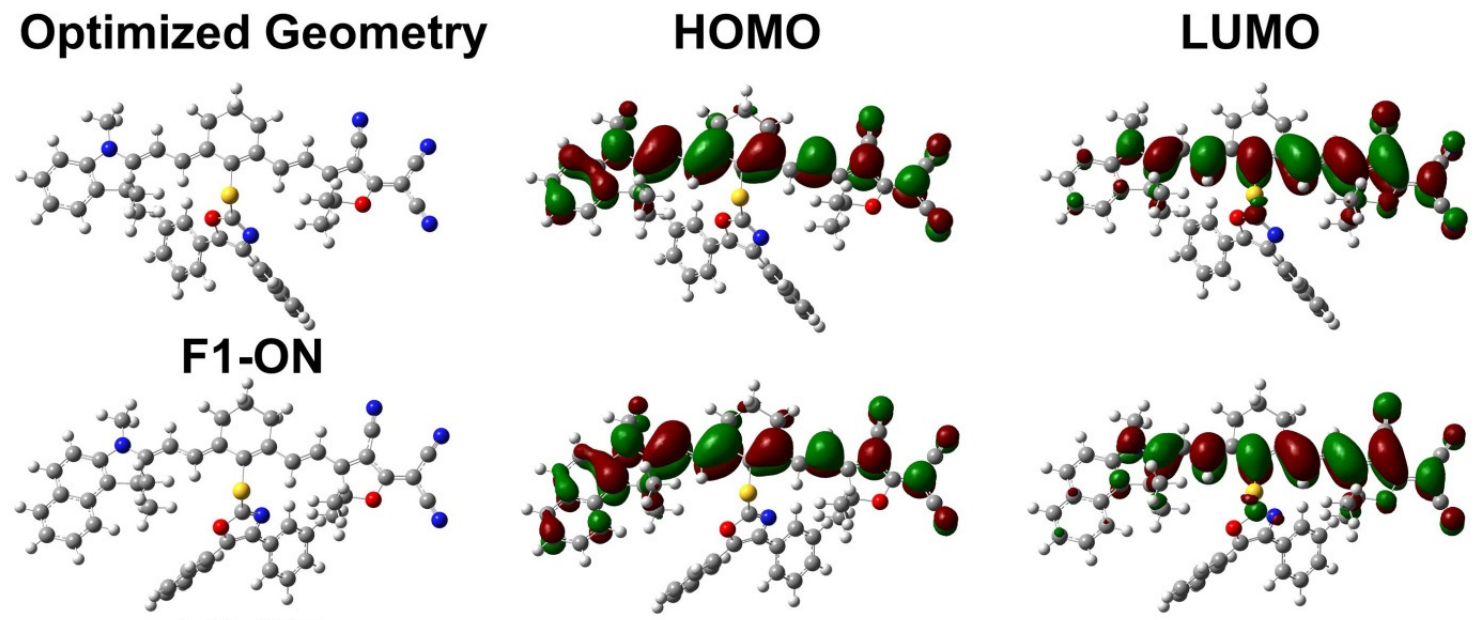

F2-ON

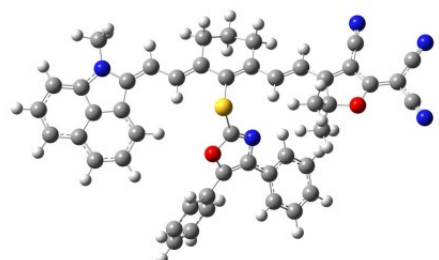

F3-ON
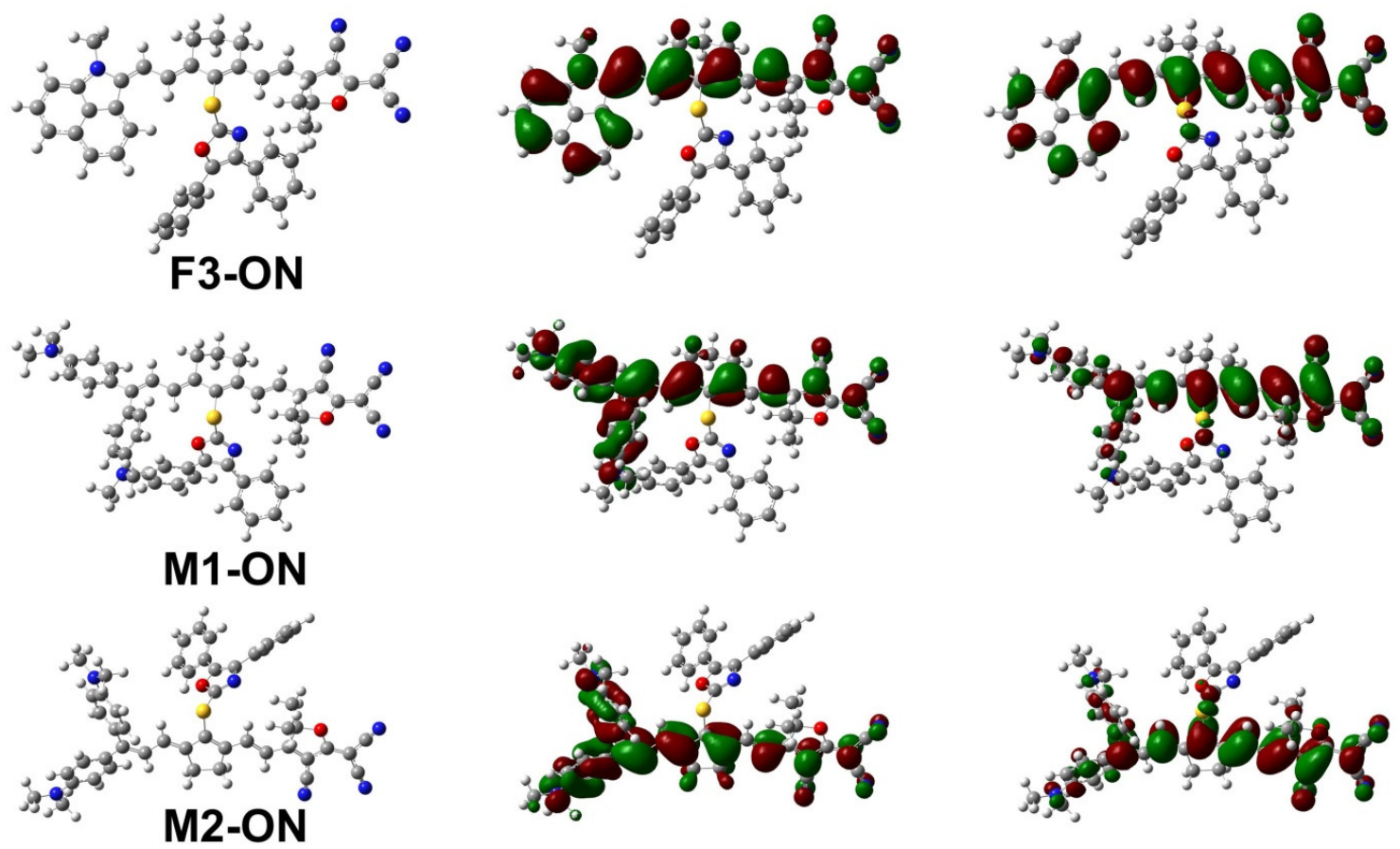

Fig. S7 Optimized geometry, HOMO and LUMO of chromophores from DFT calculation. 
Table S2 The calculated dipole moment and BLA of chromophores in gas phase.

\begin{tabular}{cccccc}
\hline Chromophore & $\mu_{\mathrm{x}}(\mathrm{D})$ & $\mu_{\mathrm{y}}(\mathrm{D})$ & $\mu_{\mathrm{z}}(\mathrm{D})$ & $\mu_{\text {total }}(\mathrm{D})$ & $\mathrm{BLA}(\AA)^{*}$ \\
\hline F1-ON & -23.8009 & 2.3742 & -0.4271 & 23.9229 & $0.0480(0.0465)$ \\
F2-ON & 24.6112 & 2.0807 & -2.3518 & 24.8107 & $0.0486(0.0453)$ \\
F3-ON & -22.3733 & 1.6541 & 0.8022 & 22.4488 & $0.0477(0.0433)$ \\
M1-ON & 26.4579 & 5.8771 & 0.9863 & 27.1208 & $0.0483(0.0435)$ \\
M2-ON & -24.1973 & 8.9671 & 0.2655 & 25.8067 & $0.0433(0.0407)$ \\
\hline
\end{tabular}

* The BLA of chloro-substituted chromophores F1, F2, F3, M1 and M2 have been added to the parentheses for comparison, respectively.

Table S3 The calculated dipole moments and BLA of chromophores in chloroform.

\begin{tabular}{cccccc}
\hline Chromophore & $\mu_{\mathrm{x}}(\mathrm{D})$ & $\mu_{\mathrm{y}}(\mathrm{D})$ & $\mu_{\mathrm{z}}(\mathrm{D})$ & $\mu_{\text {total }}(\mathrm{D})$ & BLA $(\AA)$ \\
\hline F1-ON & -35.2032 & 2.1093 & -0.7752 & 35.2748 & $0.0227(0.0224)$ \\
F2-ON & -35.5477 & 0.9481 & 3.3173 & 35.7147 & $0.0247(0.0207)$ \\
F3-ON & -32.7495 & 0.8645 & 1.3949 & 32.7906 & $0.0248(0.0233)$ \\
M1-ON & 37.9212 & 5.0572 & 2.5426 & 38.3413 & $0.0215(0.0205)$ \\
M2-ON & 35.6647 & 11.9869 & 0.0409 & 37.6252 & $0.0195(0.0193)$ \\
\hline
\end{tabular}

* The BLA of chloro-substituted chromophores F1, F2, F3, M1 and M2 have been added to the parentheses for comparison, respectively.

Table S4 The HOMO/LUMO analysis by DFT and CV and the comparison with photo-physical properties of push-pull heptamethine chromophores.

\begin{tabular}{c|ccc|ccc|cccc}
\hline & \multicolumn{3}{|c|}{ DFT } & \multicolumn{3}{c|}{ CV } & \multicolumn{4}{c}{ UV-vis-NIR } \\
\cline { 2 - 11 } Chromophore & $\begin{array}{c}\text { HOMO } \\
\mathrm{eV}\end{array}$ & $\begin{array}{c}\mathrm{eV} \\
\mathrm{eV}\end{array}$ & $\begin{array}{c}E_{\mathrm{g}} \\
\mathrm{eV}\end{array}$ & $\begin{array}{c}\text { HOMO } \\
\mathrm{eV}\end{array}$ & $\begin{array}{c}\text { LUMO } \\
\mathrm{eV}\end{array}$ & $\begin{array}{c}E_{\mathrm{g}} \\
\mathrm{eV}\end{array}$ & $\begin{array}{c}\lambda_{\max }\left(\mathrm{CHCl}_{3}\right) \\
\mathrm{nm}\end{array}$ & $\begin{array}{c}\lambda_{\max }(\text { film }) \\
\mathrm{nm}\end{array}$ & $\begin{array}{c}\lambda_{\text {onset }}(\text { film }) \\
\mathrm{nm}\end{array}$ & $\begin{array}{c}E_{\mathrm{g}} \\
\mathrm{eV}\end{array}$ \\
\hline F1-ON & -5.11 & -3.05 & 2.06 & -4.87 & -3.69 & 1.18 & 811 & 919 & 1030 & 1.20 \\
F2-ON & -5.05 & -3.02 & 2.03 & -4.84 & -3.68 & 1.16 & 846 & 940 & 1050 & 1.18 \\
F3-ON & -5.09 & -3.23 & 1.86 & -4.93 & -3.83 & 1.10 & 829 & 890 & 1168 & 1.06 \\
M1-ON & -4.95 & -3.03 & 1.92 & -4.85 & -3.81 & 1.05 & 795 & 824 & 1155 & 1.07 \\
M2-ON & -4.86 & -3.07 & 1.79 & -4.85 & -3.84 & 1.01 & 830 & 855 & 1231 & 1.01 \\
\hline
\end{tabular}


Hydrogen bond geometry of F3 and F3-ON chromophores

Table S5 Hydrogen bond geometry of $\mathbf{F 3}\left(\AA,{ }^{\circ}\right)$.

\begin{tabular}{lllll}
\hline $\mathrm{D}-\mathrm{H} \cdots \mathrm{A}$ & $\mathrm{D}-\mathrm{H}$ & $\mathrm{H} \cdots \mathrm{A}$ & $\mathrm{D} \cdots \mathrm{A}$ & $\mathrm{D}-\mathrm{H} \cdots \mathrm{A}$ \\
\hline $\mathrm{C} 10-\mathrm{H} 10 \cdots \mathrm{N} 4$ & 0.940 & 2.673 & 3.477 & 143.88 \\
$\mathrm{C} 14-\mathrm{H} 14 \cdots \mathrm{N} 3$ & 0.940 & 2.615 & 3.402 & 141.58 \\
\hline
\end{tabular}

Table S6 Hydrogen bond geometry of F3-ON $\left(\AA,{ }^{\circ}\right)$

\begin{tabular}{ccccc}
\hline $\mathrm{D}-\mathrm{H} \cdots \mathrm{A}$ & $\mathrm{D}-\mathrm{H}$ & $\mathrm{H} \cdots \mathrm{A}$ & $\mathrm{D} \cdots \mathrm{A}$ & $\mathrm{D}-\mathrm{H} \cdots \mathrm{A}$ \\
\hline $\mathrm{C} 44-\mathrm{H} 44 \cdots \mathrm{N} 2$ & 0.940 & 2.712 & 3.587 & 155.26 \\
$\mathrm{C} 53-\mathrm{H} 53 \mathrm{~B} \cdots \mathrm{O} 1$ & 0.970 & 2.715 & 3.682 & 175.45 \\
\hline
\end{tabular}


AFM graphs of F3/P(S-co-MMA) and F3-ON/P(S-co-MMA) films

(a)

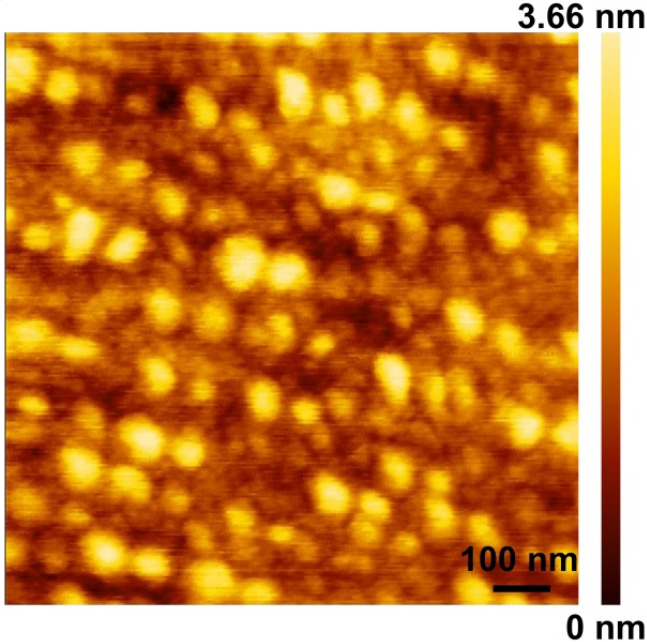

(b)

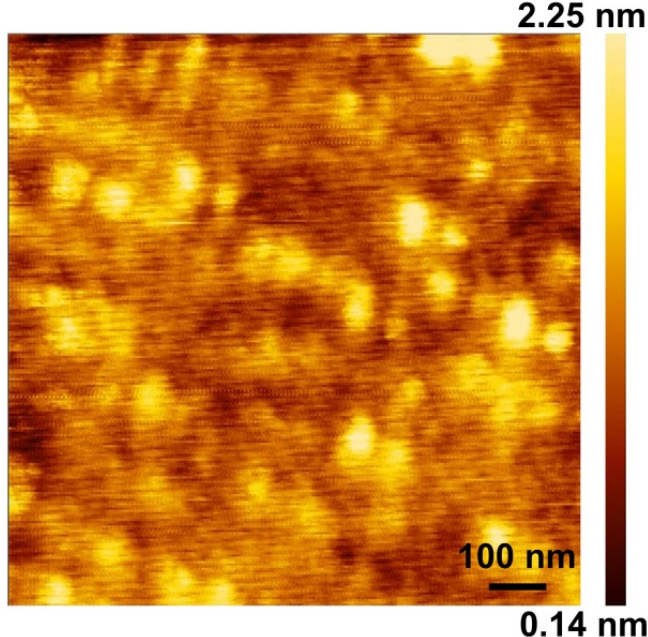

Figure. S8 AFM graphs of F3/P(S-co-MMA) (a) and F3-ON/ P(S-co-MMA) (b). 


\section{NMR and HRMS Spectra of chromophores}

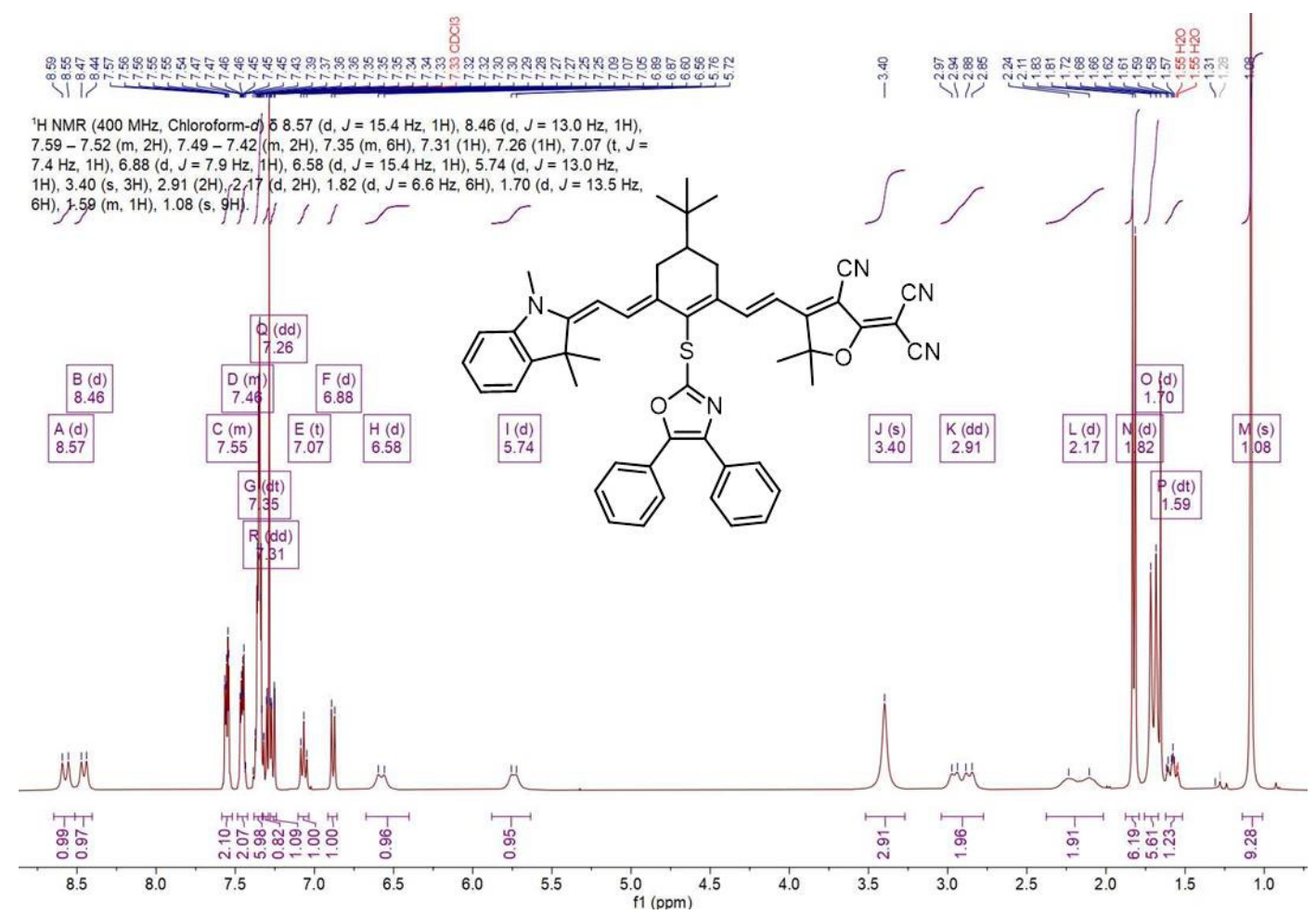

Fig. S9 ${ }^{1} \mathrm{H}$ NMR spectra of F1-ON.
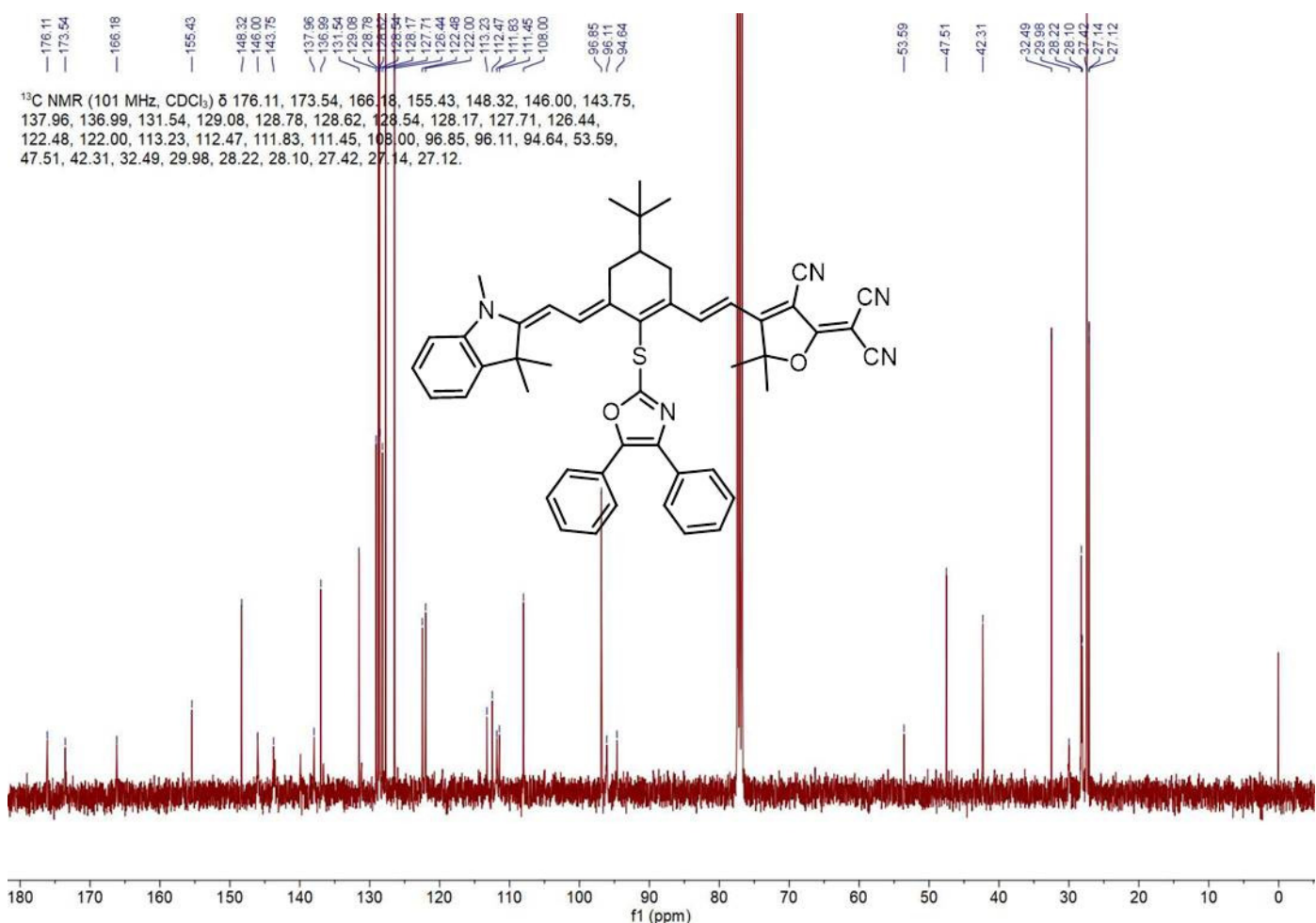

Fig. S10 ${ }^{13} \mathrm{C}$ NMR spectra of F1-ON. 


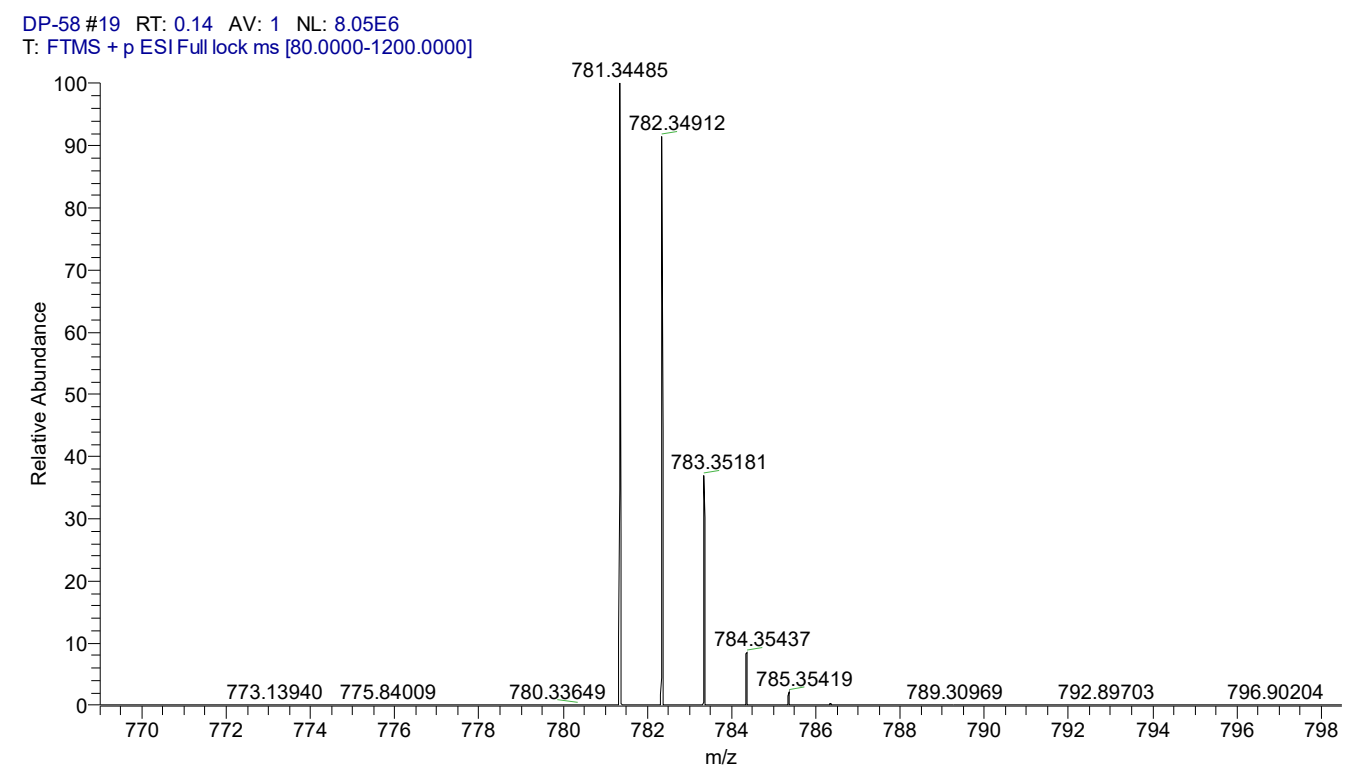

Fig. S11 HRMS spectrum of F1-ON.

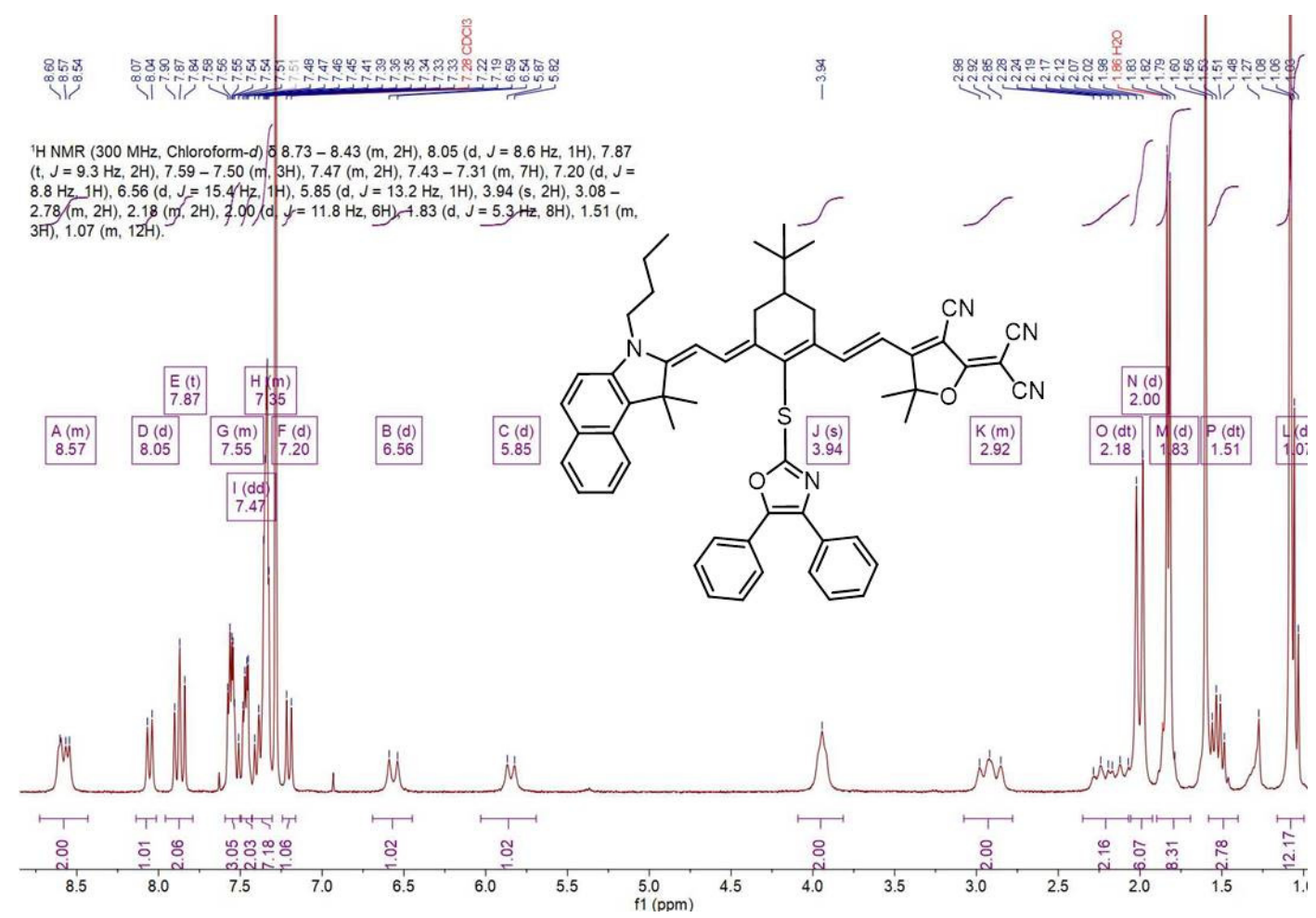

Fig. S12 ${ }^{1} \mathrm{H}$ NMR spectra of F2-ON. 


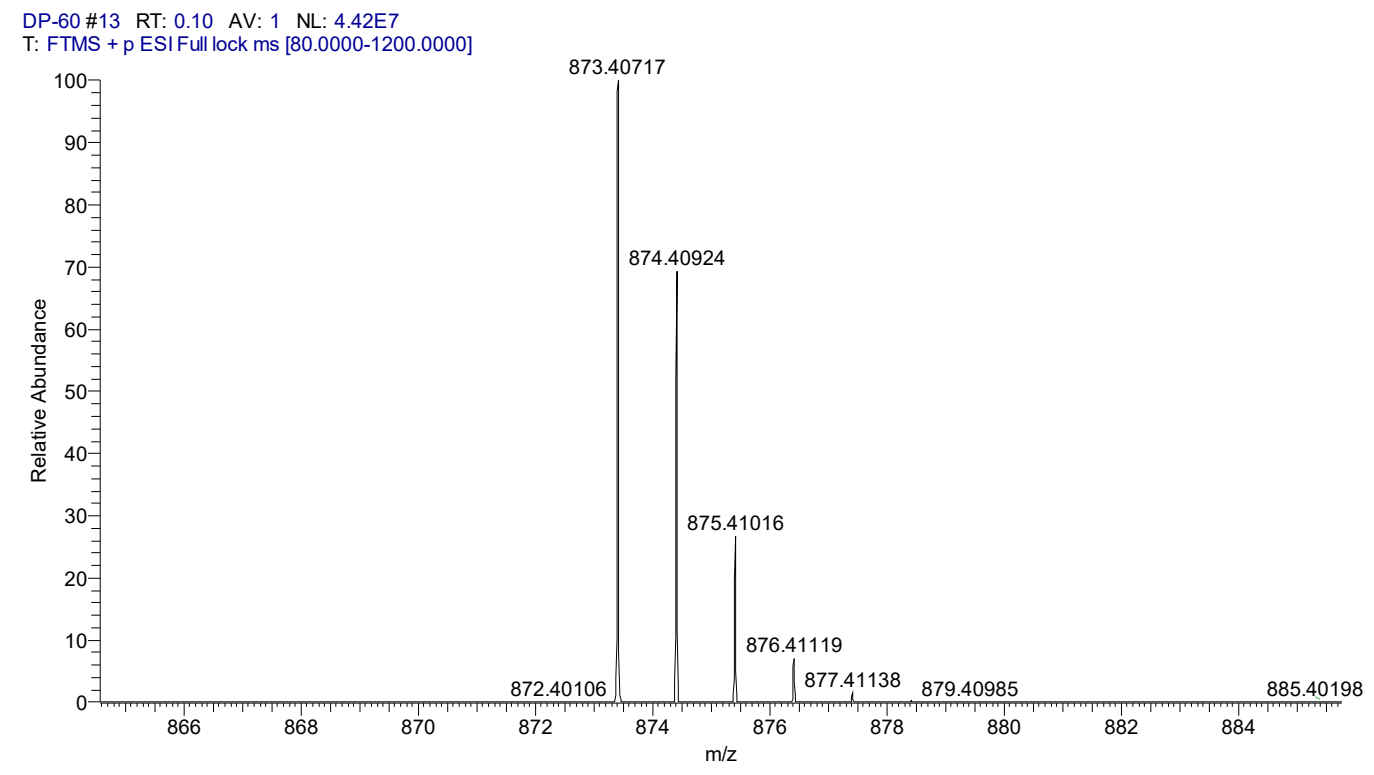

Fig. S13 HRMS spectrum of F2-ON.

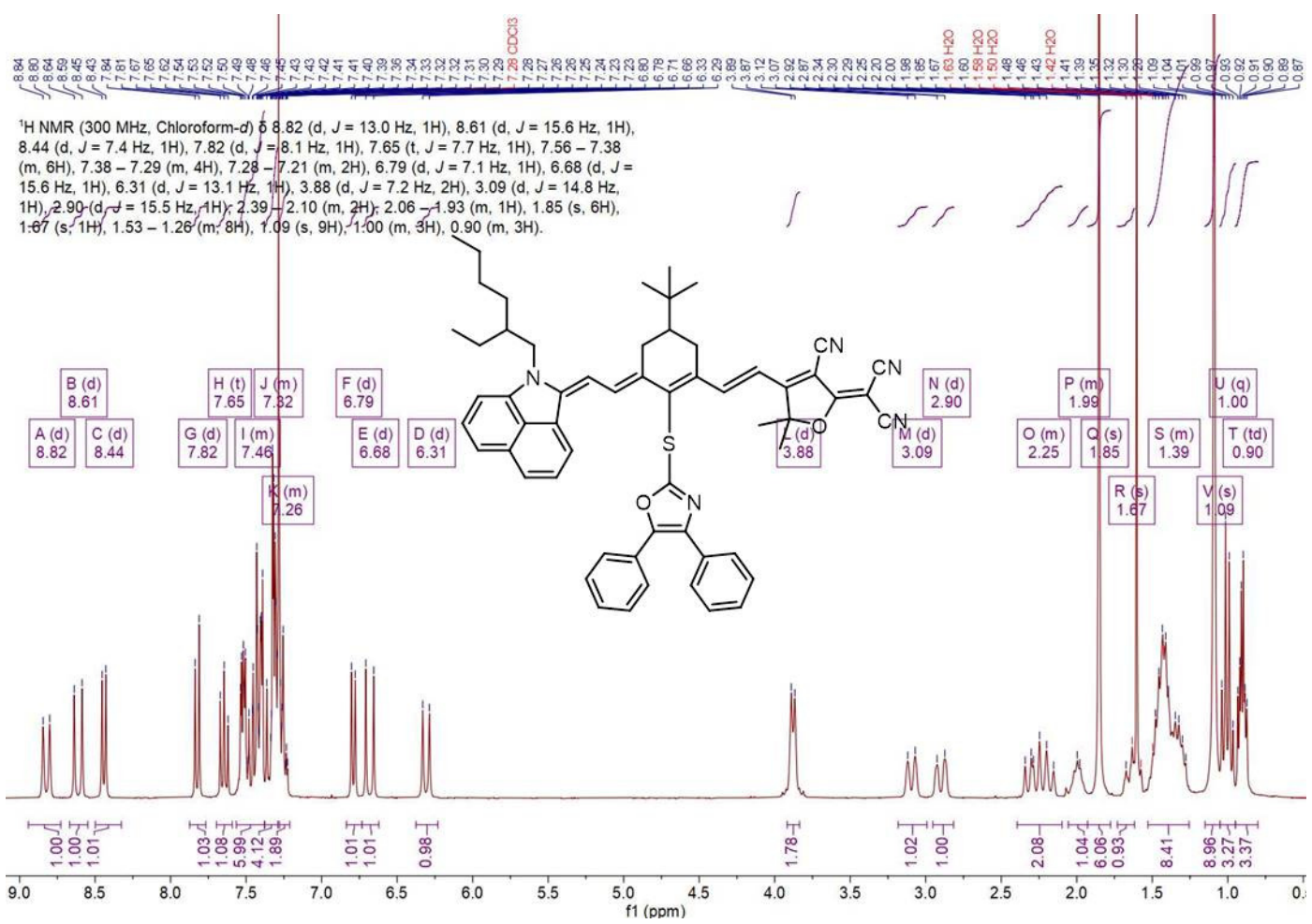

Fig. S14 ${ }^{1}$ H NMR spectra of F3-ON. 

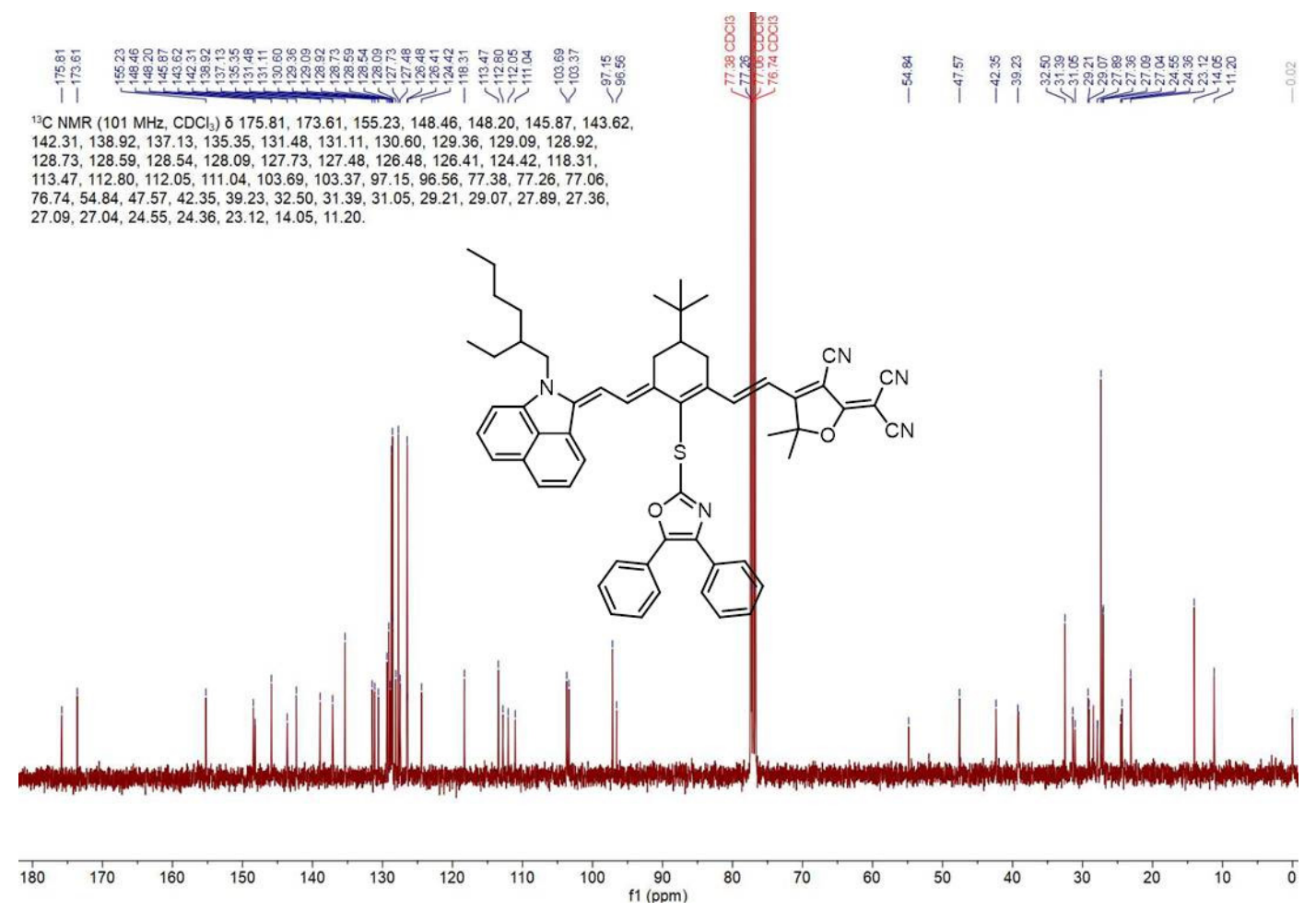

Fig. S15 ${ }^{13} \mathrm{C}$ NMR spectra of F3-ON.

DP-61 \#12 RT: 0.09 AV: 1 NL: 2.74E7

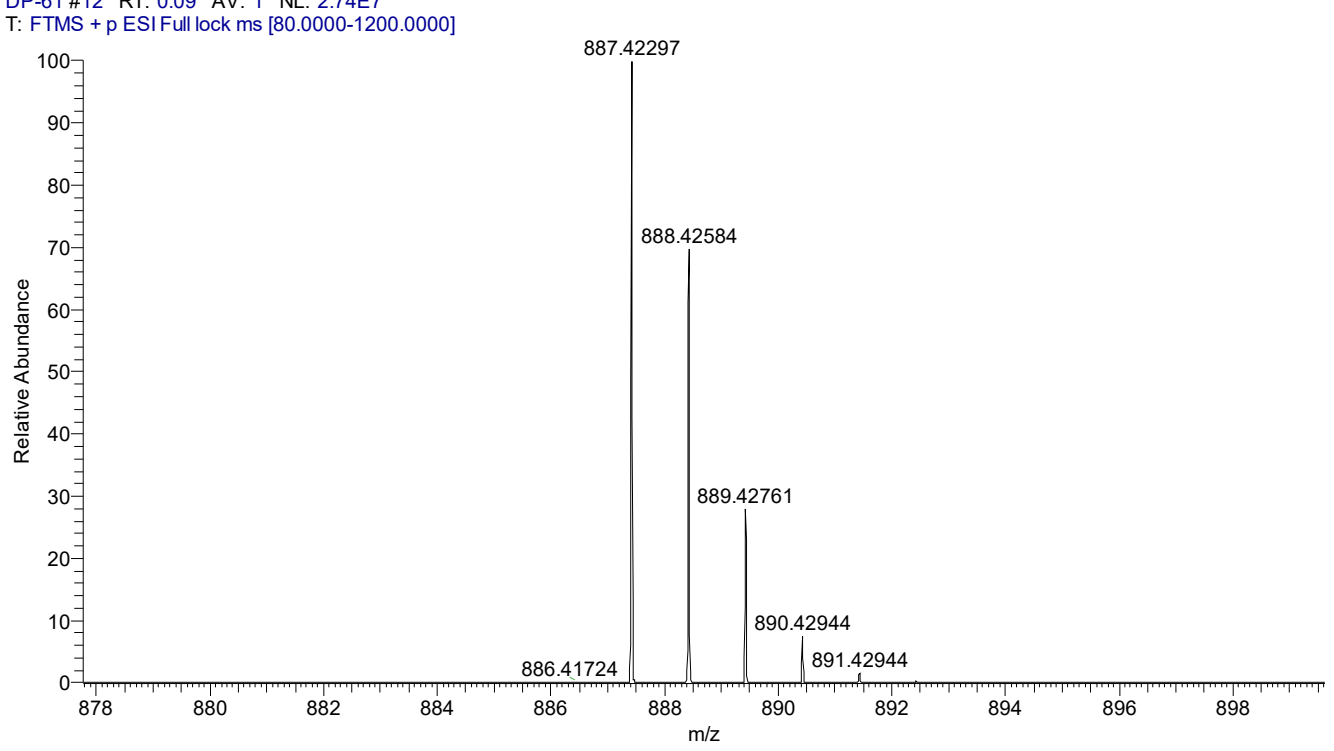

Fig. S16 HRMS spectrum of F3-ON. 


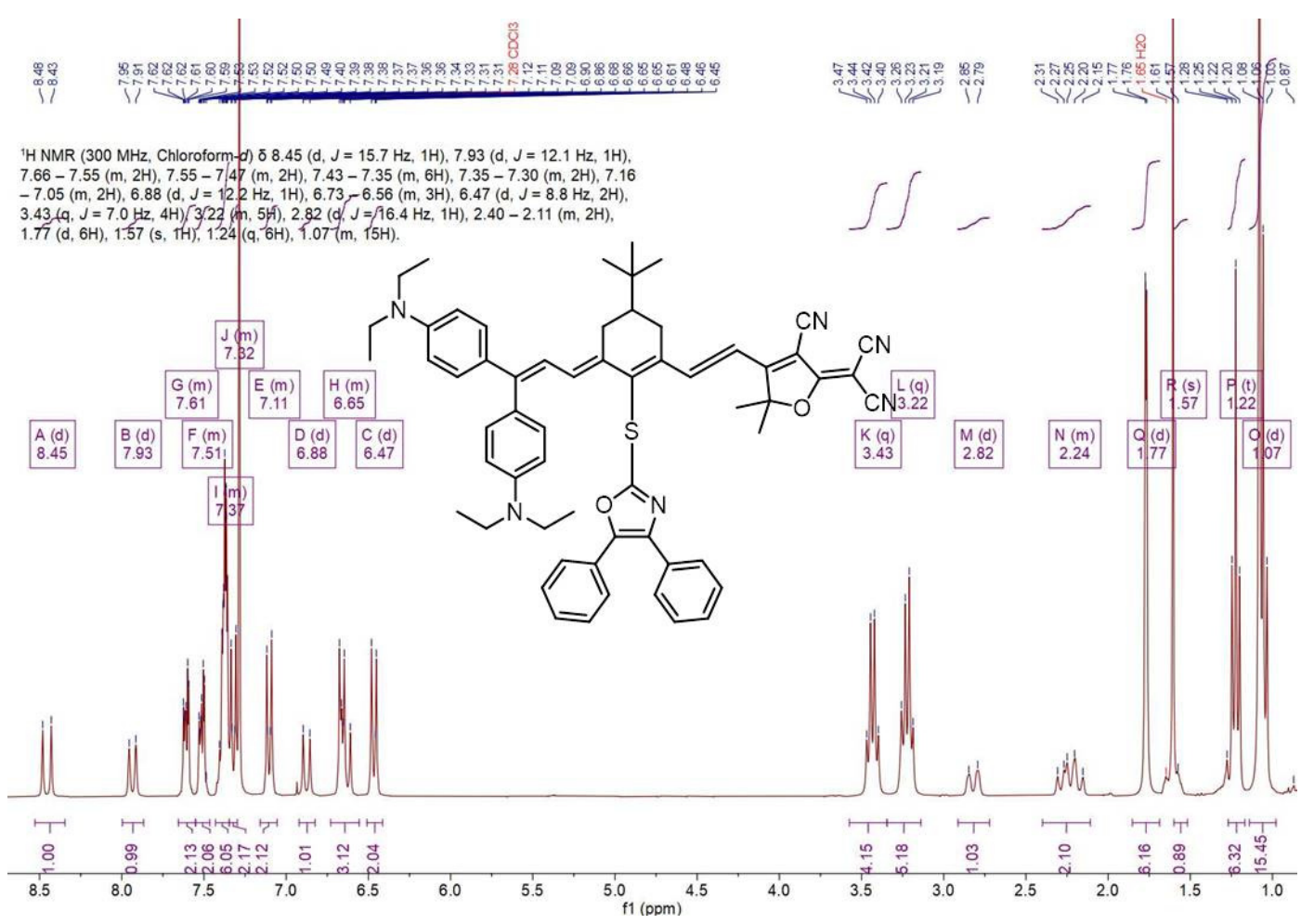

Fig. S17 ${ }^{1} \mathrm{H}$ NMR of M1-ON.

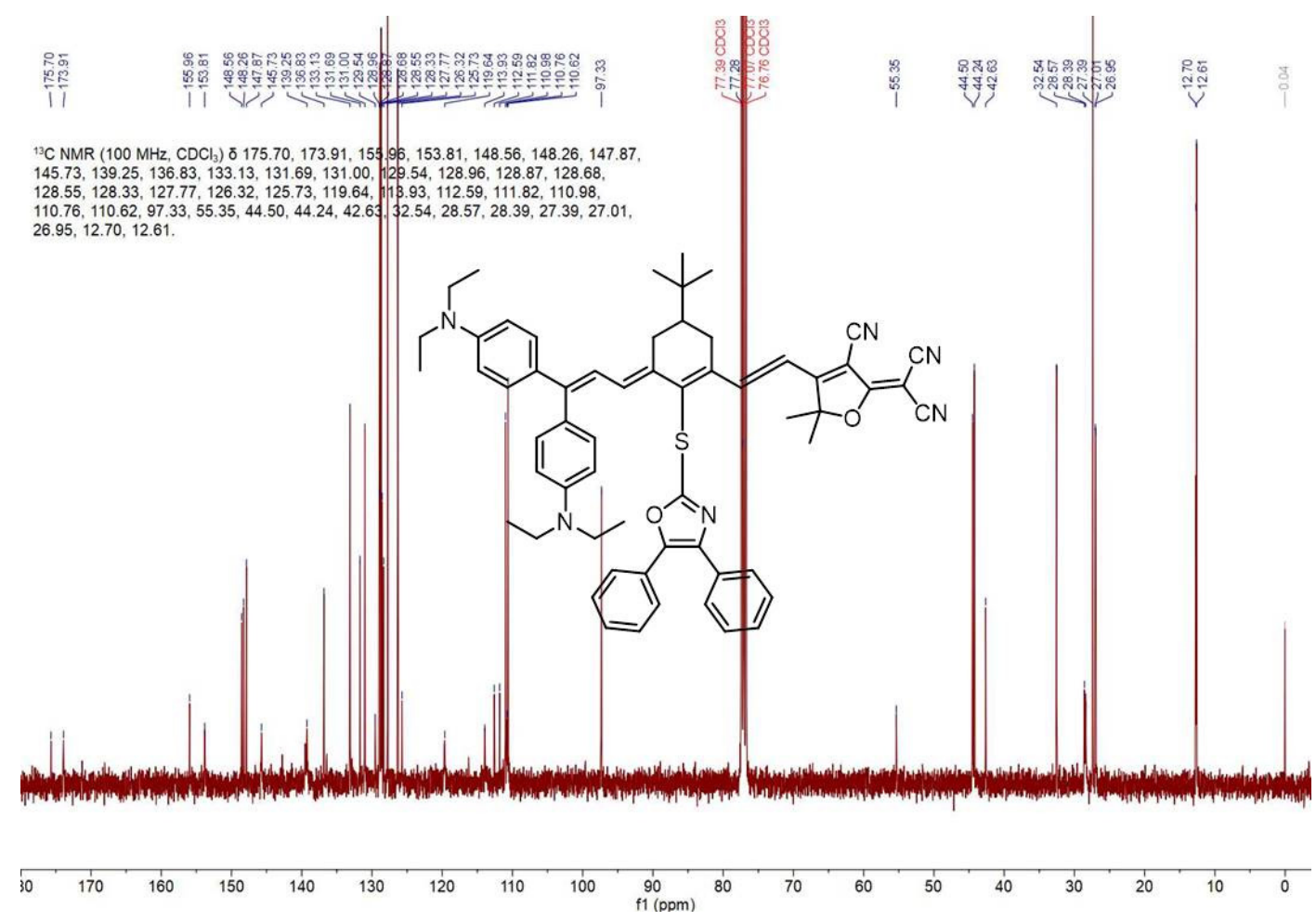

Fig. S18 ${ }^{13} \mathrm{C}$ NMR of M1-ON. 


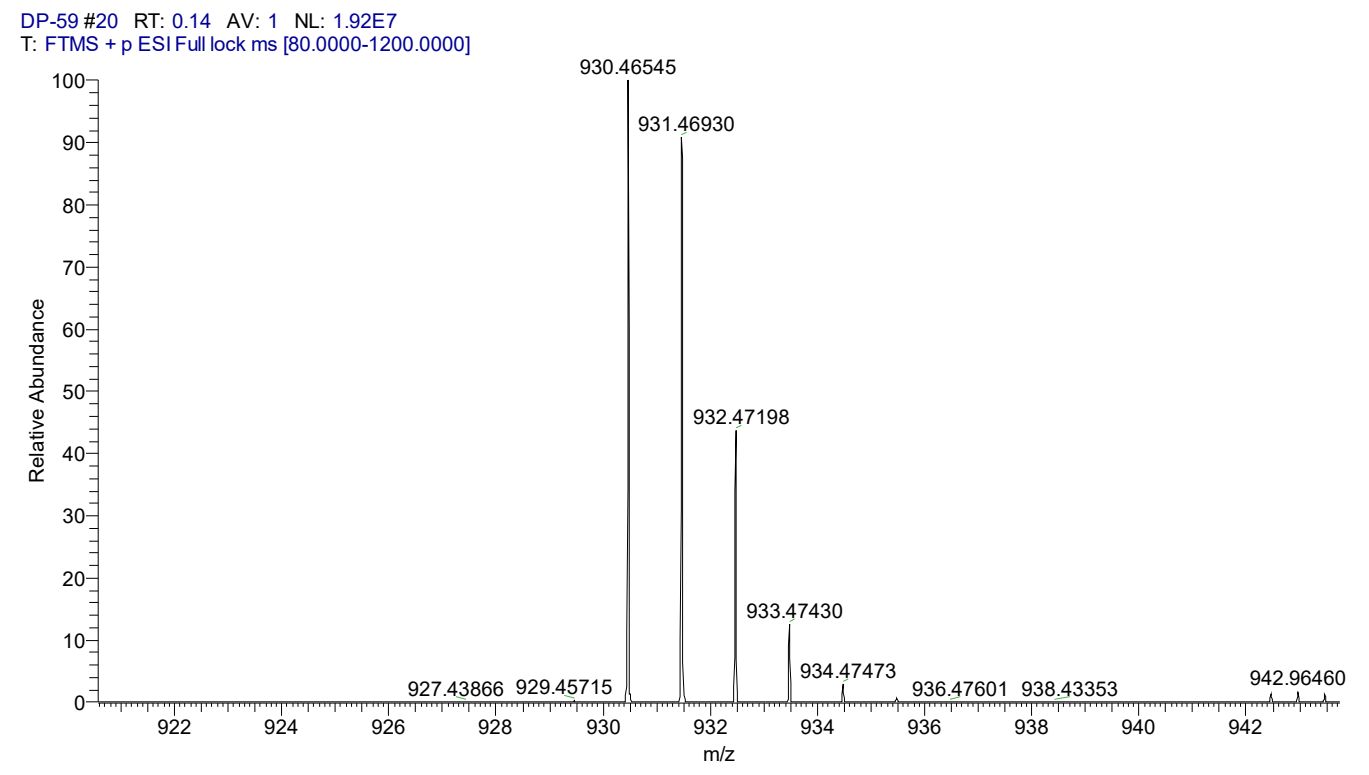

Fig. S19 HRMS spectrum of M1-ON.

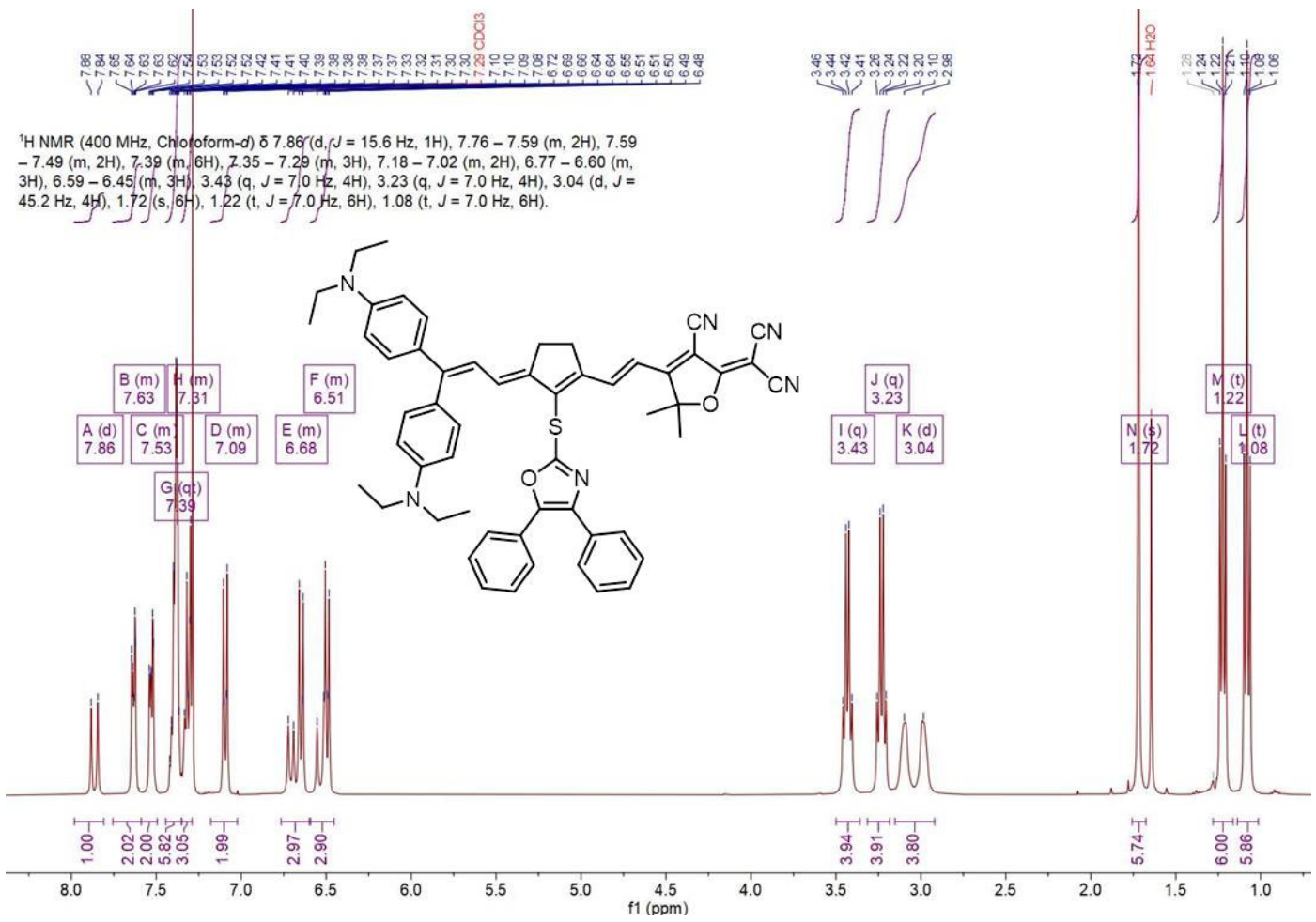

Fig. S20 ${ }^{1} \mathrm{H}$ NMR of M2-ON. 
${ }^{13} \mathrm{C} \mathrm{NMR}\left(100 \mathrm{MHz}, \mathrm{CDCl}_{3}\right)$ б $175.53,172.64,154.64,152.00,148.46,148.23,148.13$ $146.05,144.52,138.56,136.96,132.87,131.53,131.39,130.81,129.51,129.08$,

$111.75,111.00,110.80,110.59,97.57,97.04,55.56,44.50,44.17,29.21,27.19,26.90$ $12.70,12.64$
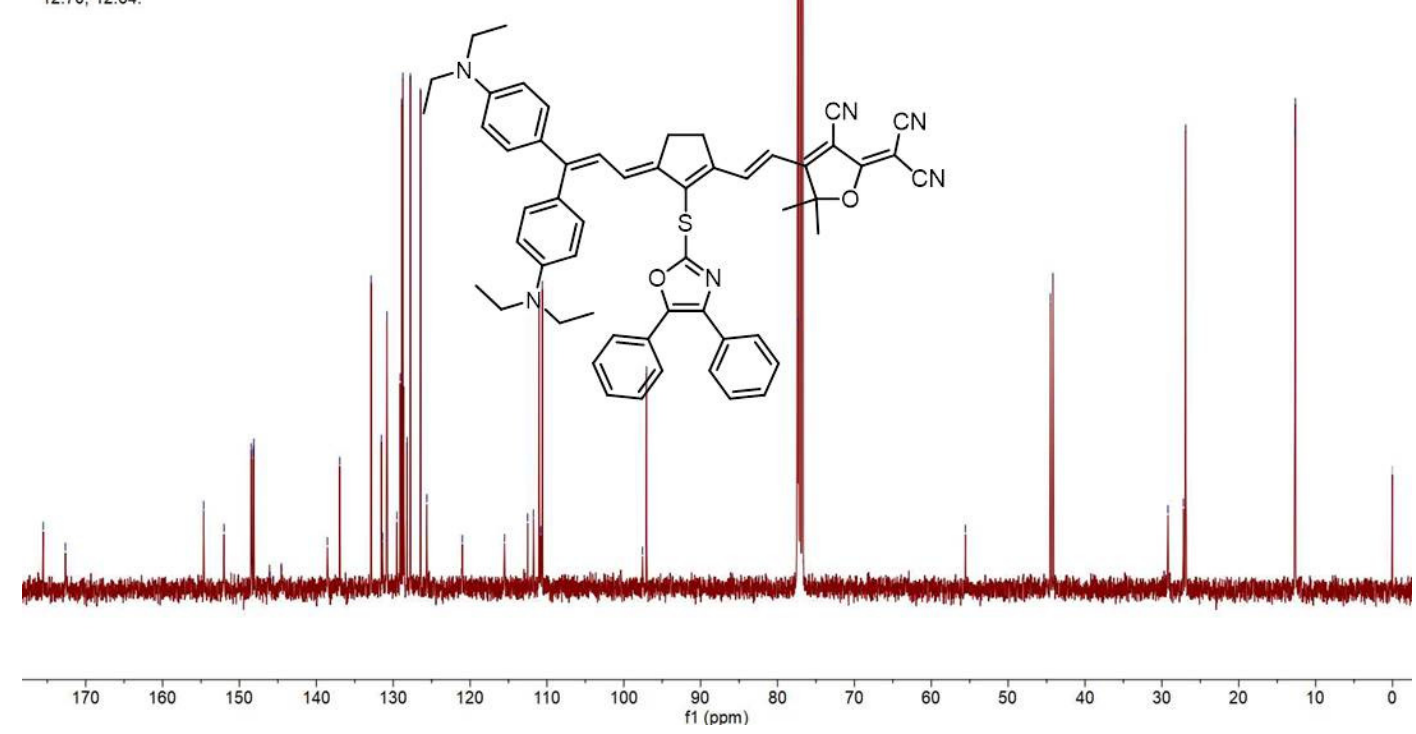

Fig. S21 ${ }^{13} \mathrm{C}$ NMR of M2-ON.

DP-57 \#26 RT: 0.17 AV: 1 NL: 3.83E4

$\mathrm{T}:$ FTMS + p ESI Full lock ms [80.0000-1200.0000]

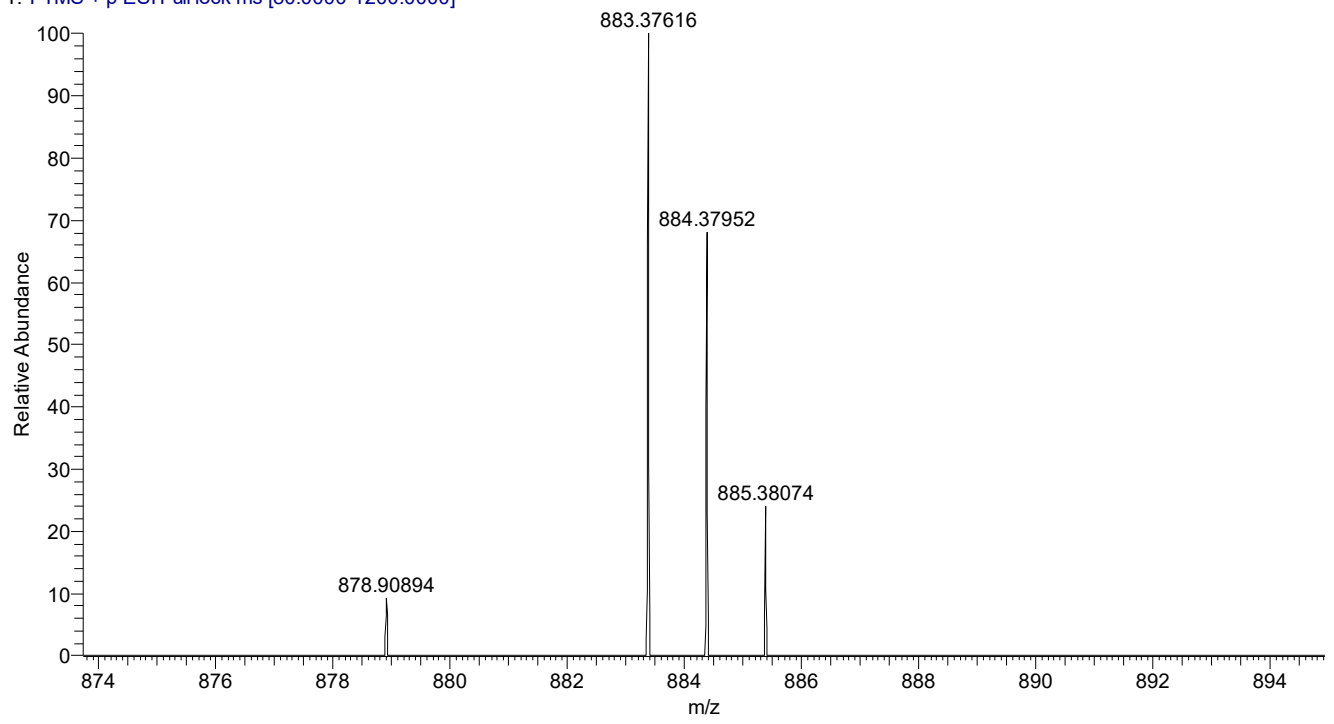

Fig. S22 HRMS spectrum of M2-ON. 
Thermogravimetric analyses of chromophores

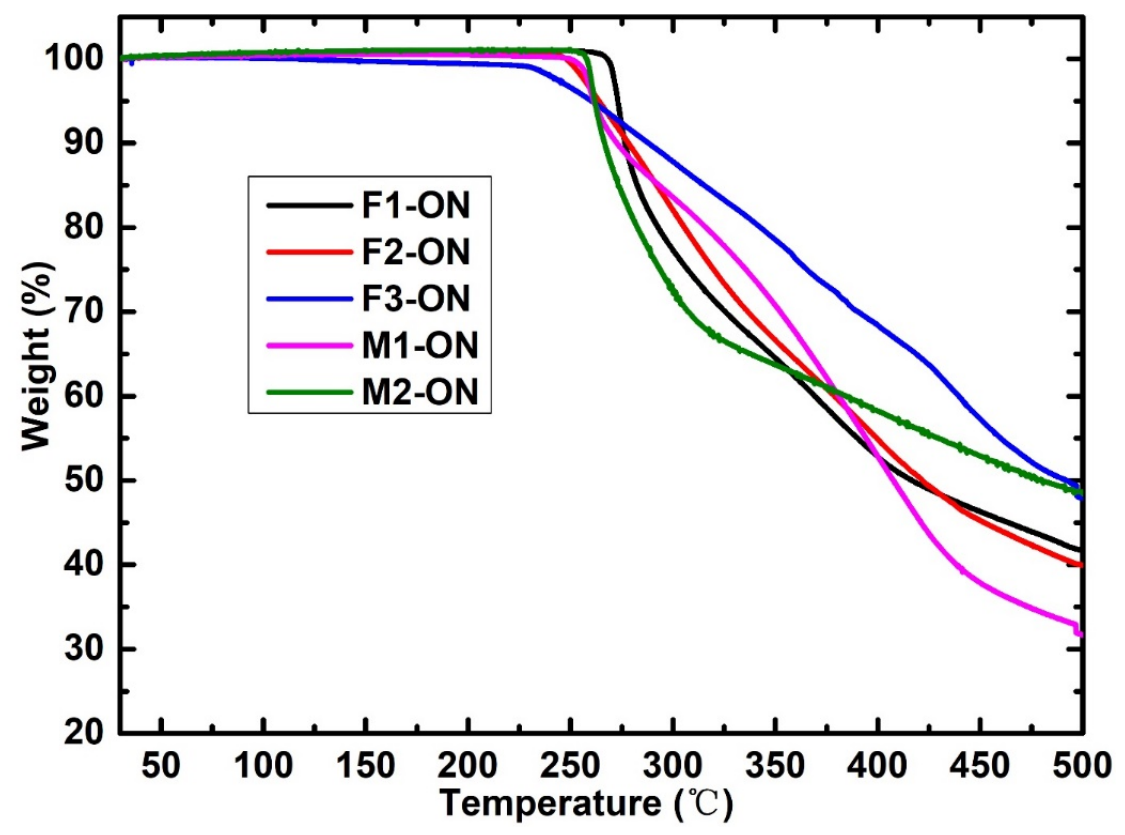

Fig. S23 TGA of chromophores. 
Analysis of order parameters by ROGM

The order parameters of poled films were calculated from refractive indices of $n_{T E}$ and $n_{T M}$, and used as a measure of chromophore alignment in the poled films. A Sellmeier fit of refractive indices was conducted on values of refractive indices at $1304 \mathrm{~nm}$ and $1541 \mathrm{~nm}$ using a simple model of absorption and dispersion, in which the refractive index $(n)$ is assumed to have contributions from both a non-resonant background $n_{0}$ and the two-level charge transfer resonance at frequency $v_{0}$ in $\mathrm{cm}^{-1}$.

Thus at an operational frequency of $v$, we have $n=n_{0}+\frac{A}{v_{0}^{2}-v^{2}}$, and the order parameter $\Phi$ for poled EO films is given by $\Phi=\frac{\delta_{T M}-\delta_{T E}}{\delta_{T M}+2 \delta_{T E}}$, where $\delta_{T M}=n_{T M}-n_{O}$ and $\delta_{T E}=n_{T E}-n_{O}$. We then correlate the order parameter $\Phi$ from the birefringence measurement to the polar order parameter $\left\langle\cos ^{3} \theta\right\rangle$ of poled films according to the second and third Legendre polynomials as a function of $\mu \mathrm{F} / \mathrm{kT}$ according to Figure $\mathrm{S} 24$.

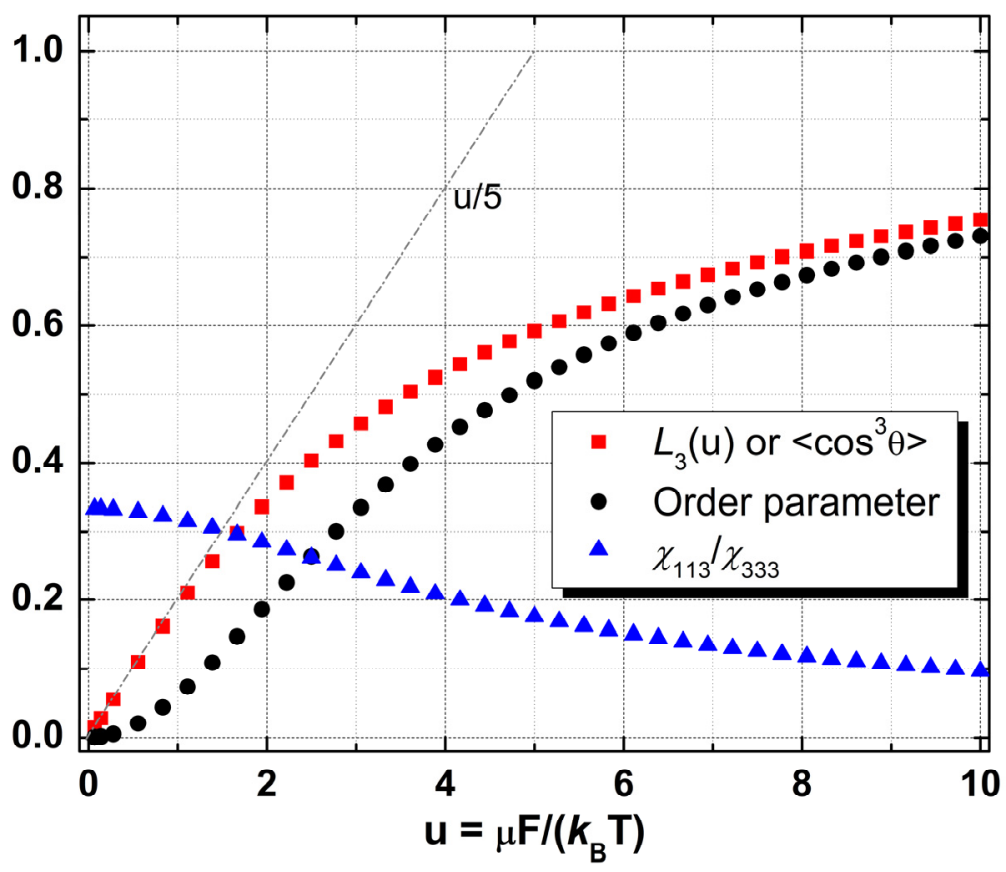

Figure S24. The order parameter and third Legendre polynomial as a function of $u$, where $u=$ $\mu \mathrm{F} / \mathrm{kT}$. Reproduced with permission from Burland, D. M.; Miller, R. D.; Walsh, C. A., Second-order nonlinearity in poled-polymer systems, Chem. Rev., 1994, 94: 31-75. Copyright (1994) American Chemical Society. 


\section{Reference:}

\section{Gaussian 09, Revision B.01,}

M. J. Frisch, G. W. Trucks, H. B. Schlegel, G. E. Scuseria, M. A. Robb, J. R. Cheeseman, G. Scalmani, V. Barone, B. Mennucci, G. A. Petersson, H. Nakatsuji, M. Caricato, X. Li, H. P. Hratchian, A. F. Izmaylov, J. Bloino, G. Zheng, J. L. Sonnenberg, M. Hada, M. Ehara, K. Toyota, R. Fukuda, J. Hasegawa, M. Ishida, T. Nakajima, Y. Honda, O. Kitao, H. Nakai, T. Vreven, J. A. Montgomery, Jr., J. E. Peralta, F. Ogliaro, M. Bearpark, J. J. Heyd, E. Brothers, K. N. Kudin, V. N. Staroverov, T. Keith, R. Kobayashi, J. Normand, K. Raghavachari, A. Rendell, J. C. Burant, S. S. Iyengar, J. Tomasi, M. Cossi, N. Rega, J. M. Millam, M. Klene, J. E. Knox, J. B. Cross, V. Bakken, C. Adamo, J. Jaramillo, R. Gomperts, R. E. Stratmann, O. Yazyev, A. J. Austin, R. Cammi, C. Pomelli, J. W. Ochterski, R. L. Martin, K. Morokuma, V. G. Zakrzewski, G. A. Voth, P. Salvador, J. J. Dannenberg, S. Dapprich, A. D. Daniels, O. Farkas, J. B. Foresman, J. V. Ortiz, J. Cioslowski, and D. J. Fox, Gaussian, Inc., Wallingford CT, 2010. 